\title{
Deciphering clonality in aneuploid breast tumors using SNP array and sequencing data
}

\author{
Ingrid M Lönnstedt ${ }^{1,2,3^{*}}$, Franco Caramia ${ }^{3}$, Jason $\mathrm{Li}^{3}$, Debora Fumagalli ${ }^{4}$, Roberto Salgado ${ }^{4}$, Andrew Rowann, \\ Max Salm ${ }^{6}$, Nnennaya Kanu ${ }^{7}$, Peter Savas ${ }^{3}$, Stuart Horswell ${ }^{6}$, Stephan Gade ${ }^{8}$, Sibylle Loibl ${ }^{8}$, Patrick Neven $^{9}$, \\ Christos Sotiriou ${ }^{4}$, Charles Swanton ${ }^{5,10}$, Sherene Loi ${ }^{2,3^{*}}$ and Terence P Speed ${ }^{1,11}$
}

\begin{abstract}
Intra-tumor heterogeneity concerns the existence of genetically different subclones within the same tumor. Single sample quantification of heterogeneity relies on precise determination of chromosomal copy numbers throughout the genome, and an assessment of whether identified mutation variant allele fractions match clonal or subclonal copy numbers. We discuss these issues using data from SNP arrays, whole exome sequencing and pathologist purity estimates on several breast cancers characterized by ERBB2 amplification. We show that chromosomal copy numbers can only be estimated from SNP array signals or sequencing depths for subclonal tumor samples with simple subclonal architectures under certain assumptions.
\end{abstract}

\section{Background}

Genomes can vary between cells within a tumor. Mutations and copy number $(\mathrm{CN})$ alterations which appear during tumor development result in genomic subclones emerging. Subclonality of tumors is referred to as intratumor heterogeneity (ITH), a topic which has attracted much attention over the last few years [1-17]. The subclones within a tumor may display different features and respond differently to drugs. It has been speculated that heterogeneity-related endpoints - a tumor's clonal architecture, features of the subclones, or whether mutations are clonal (present equally in all tumor cells) or subclonal might serve as biomarkers for drug resistance $[5,18,19]$.

Heterogeneity of cancers has been studied by comparing mutations and $\mathrm{CN}$ alterations between spatially separated $[3,6,7]$ or sequential [10] samples from the same tumor, or between primary and secondary tumors [11] from the same patient.

To meet clinical demand, recent studies have attempted to assess heterogeneity from single tumor samples based on whole genome sequencing (WGS) $[4,8,9,12,14-16]$ or the cheaper whole exome sequencing (WES) $[1,2,5,13,17]$,

\footnotetext{
* Correspondence: ingrid.lonnstedt@gmail.com; sherene.loi@petermac.org

${ }^{1}$ Bioinformatics Division, The Walter and Eliza Hall Institute of Medical

Research, 1G Royal Parade, Parkville, VIC 3052, Australia

${ }^{2}$ University of Melbourne, Melbourne, VIC 3010, Australia

Full list of author information is available at the end of the article
}

usually in combination with genome-wide data from SNP arrays. In general, the average $\mathrm{CN}$ across all cells in the tumor sample is estimated at numerous genomic positions from SNP arrays or sequencing data, and these values are joined up into genome segments of constant $\mathrm{CN}$ (from now on called segmented $\mathrm{CN}$ data). Next, the variant allele fraction (VAF) of each somatic mutation identified in the sequencing data is compared to the local $\mathrm{CN}$ estimate, in order to classify the mutation as clonal or subclonal. Some papers proceed to construct a phylogenetic tree which visualizes the subclonal evolution of the tumor $[8,9,14-17]$.

We have looked at 52 single samples from newly diagnosed HER2-positive breast cancer tumors in the RESPONSIFY project [20] using Affymetrix SNP 6.0 arrays, WES and pathologist purity estimates. Our tumors all show heterogeneity, in that most are highly aneuploid throughout most of the genome in only a fraction of the cells. The scientific question driving this methodology project was whether identified mutations are clonal or subclonal. In particular, we hoped to assess clonality of specific $\mathrm{CN}$ alterations, such as those of HER2, by inferring the status of mutations present at their genomic location. It turns out, as we will demonstrate, that classification of mutations in 
samples with heterogeneity is not always possible with the data we had.

The focus of this paper is on the stages of analysis preceding automatic approaches which take input data and return an estimated clonality status of each mutation. Our principal aim is to highlight challenges in $\mathrm{CN}$ estimation infrequently acknowledged in the literature which influence mutation classification. We also propose solutions that may aid in the quantification of ITH in tumor samples that have high levels of $\mathrm{CN}$ alteration. Such a method will help in understanding how ITH is related to prognosis, that is, survival of patients diagnosed with breast cancers, as well as drug resistance, as it will be applicable to large datasets with annotated treatment and clinical outcome information.

We make extensive use of grid plots, which help visualize the clonal architecture of aneuploid tumor samples and provide visual feedback on the absence or presence of bias in segmented $\mathrm{CN}$ data. We also describe the key issues and challenges in $\mathrm{CN}$ estimation of subclonal samples, and show how local subclonal integer $\mathrm{CN}$ estimates are vital for correct classification of mutations.

Our demonstrations are restricted to a handful of the 52 RESPONSIFY HER2-positive breast cancer samples. Complete analyses of all samples with medical results, including potential biomarkers for resistance to trastuzumabbased therapy, will be published separately.

Our results are divided into three parts (A to $\mathrm{C}$ ). In part A we present grid plots and demonstrate key issues in the estimation of $\mathrm{CN}$ of subclonal tumor samples in a simulated setting, to show that even with no noise or bias, subclonal chromosomal CNs can only be estimated in some genome segments, in samples with simple subclonal architectures, and even then relying on subjective assumptions. In part B, still in a simulated framework with no noise or bias, we show how the subclonal chromosomal CNs play a vital role in the classification of mutations as clonal or subclonal. In part $\mathrm{C}$ we briefly discuss our data. We suggest a probabilistic strategy to separate subclonality from noise in segmented $\mathrm{CN}$ data, and to assign a clonality status to a mutation. We also supply a two-dimensional grid rotation method to adjust for B allele fraction bias, which is common in our datasets.

We will refer to the number of chromosomal copies at a genome position in specified cells as their (true) integer $\mathrm{CN}$. The average integer $\mathrm{CN}$ across cells from a tumor sample at a genome position will be called the (true) average CN. SNP array signals, which have been preprocessed, segmented and possibly normalized towards germline array data so that they are supposedly proportional to average CNs apart from noise deviations, will be called array CNs. By cell fraction we mean the percentage of sample cells (out of both normal and tumor cells of a sample) that make up a specified subclone.

\section{Results}

A: Grid patterns and integer CN estimation in simulated aneuploid tumors

In this section we present grid plots and demonstrate key issues in the estimation of $\mathrm{CN}$ of subclonal tumor samples in a simulated setting, to show that even with no noise or bias, subclonal chromosomal CNs can only be estimated in some genome segments, in samples with simple subclonal architectures, and even then relying on subjective assumptions. This step is important for subsequent classification of mutations as clonal or subclonal, since the mutation VAFs depend on local integer CNs in the tumor cells.

\section{Clonal tumors and grid plots}

A normal, diploid cell has one copy of each parental chromosome in its nucleus. We say its integer $\mathrm{CNs}$ are $(1,1)$. Aneuploid tumor cells exhibit integer $\mathrm{CNs}$ other than $(1,1)$, including segments with loss of heterozygosity $(\mathrm{LOH})$, such as $(0,1)$ or $(0,2)$, or $\mathrm{CN}$ gains, such as $(1,2),(1,3)$ or $(2,2)$. Each genome segment of constant $\mathrm{CN}$ in an aneuploid tumor cell may be represented by a point in a grid plot, a figure which displays all the combinations of CNs that occur throughout the genome in that cell, in a minor (smaller) versus major (larger) homologue $\mathrm{CN}$ pattern (extending the idea of TAPS plots in [21]). Figure 1a is a grid plot of simulated integer $\mathrm{CNs}$ in a cell in which each possible major and minor combination of $0,1,2,3$ and 4 copies occurs somewhere along the genome.

Tumor samples consist of thousands of tumor cells plus an unknown fraction of normal diploid cells, which we call normal contamination. We simulate a sample
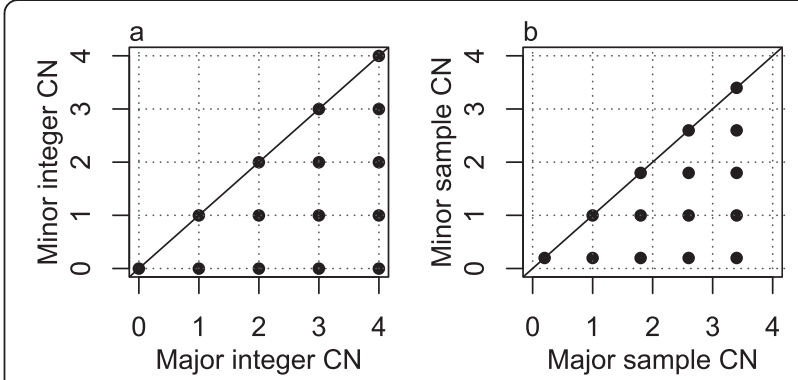

Figure 1 Grid plot of a simulated clonal tumor sample. (a) Grid plot of the integer CNs of a simulated single aneuploid tumor cell where all the combinations of major and minor integer CNs from 0 to 4 occur in the genome. (b) Grid plot of the average CNs from a simulated tumor sample with purity $80 \%$ and clonal CNs as in (a). Note how this grid is a version of (a) shrunken towards the point $(1,1)$. 
with clonal tumor CNs (identical integer CNs across all the tumor cells) as in Figure 1a with fraction (purity) $p$ of tumor cells. Each average $\mathrm{CN} e$ of a given homologue and genome segment will then have the form:

$$
e=(1-p)+p c, c=0,1,2, \ldots, 4
$$

where $c$ is the integer $\mathrm{CN}$ in the tumor cells (grid plot in Figure 1b). Compared with Figure 1a, each point in Figure $1 \mathrm{~b}$ is shifted (shrunken) towards the point $(1,1)$, since each average $\mathrm{CN}$ is the average of the integer $\mathrm{CNs}$ $(1,1)$ of the normal cells and the tumor cell integer CNs.

Integer $\mathrm{CNs}$ and the purity of a tumor sample can only be unambiguously estimated from the unbiased, noise free average CNs via Equation 1 if 1) the sample is known to be clonal, and 2) there are at least two points in the grid plot for which the difference is known on an integer $\mathrm{CN}$ scale. For example, it may be known that two consecutive vertical grid points reflect a difference of one copy in the minor homologue.

With tumor samples, it is seldom known that a sample is clonal (1), so we broaden the $\mathrm{CN}$ estimation framework to that of (potentially) subclonal tumors.

\section{Subclonal tumors}

For tumors with heterogeneity, $\mathrm{CN}$ estimation comes down to estimation of the cell fraction and integer $\mathrm{CNs}$ of each subclone. As we shall see, this is a very difficult task with the data we consider.

Grid patterns from tumor samples with heterogeneity are more complicated than those in Figure 1. We simulate a sample consisting of $20 \%$ germline cells $(p=80 \%$ purity), $\alpha=30 \%$ cells forming an aneuploid subclone A with integer CNs as in Figure $2 \mathrm{a}$ and $\beta=50 \%$ cells forming another subclone B with integer $\mathrm{CNs}$ as in Figure $2 \mathrm{~b}$.
Simulated average CNs are segment-specific averages of the subclonal integer CNs across all the sample cells, so in a given genome segment they take values of the form:

$$
e=(1-p)+\alpha c_{a}+\beta c_{b}
$$

where $\alpha+\beta=p$ and $c_{a}$ and $c_{b}$ are the integer CNs of the subclone $\mathrm{A}$ and $\mathrm{B}$ cells in that segment. The grid plot for the sample in Figure 2c consists of three small grids, each of which originates from the CNs in A combined with the CNs in one of the three B segments. The size, or rather the density, of the small grids is due to the small fraction of cells in A. The positions of the small grids follow that of a more sparse grid, determined by the larger fraction of cells in B. Alternatively, the grid plot could be seen as many three-point sparse grids (the green circles being one of them), positioned according to the denser pattern of subclone A.

For one subclone (say A), the cell fraction $(\alpha)$ can be estimated from the perfect, noise-free average CNs via Equation 2 if Condition 1: There are at least two points in the grid plot for which the difference is known (on an integer $\mathrm{CN}$ scale) and known to be due only to a change of integer $\mathrm{CNs}$ in subclone $\mathrm{A}$ (so that all other subclones have constant $\mathrm{CNs}$ throughout these two segments).

Given the cell fraction of subclone A, its integer CNs can be estimated from unbiased, noise-free average $\mathrm{CNs}$ via Equation 2 (or its extensions to more than two subclones) if Condition 2: The integer $\mathrm{CNs}$ and cell fractions of all subclones other than A of the sample are also known.

Condition 2 seems to be a catch 22 in that no subclonal integer $\mathrm{CNs}$ can be estimated without knowing the integer CNs of the other subclones, but there is an important exception. If the grid pattern suggested by
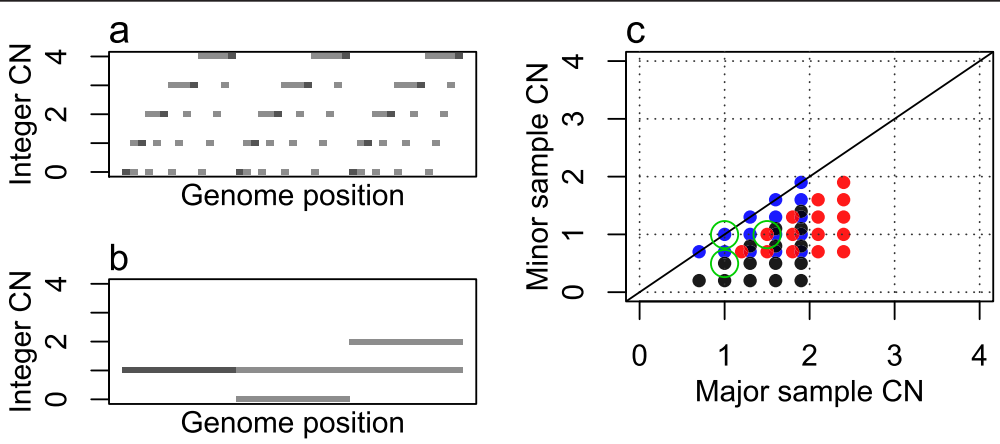

Figure 2 Grid plot of a simulated, subclonal tumor sample. (a) Genome integer CNs in the aneuploid subclone A. (b) Genome integer CNs in the less variable subclone B. (c) Grid plot from sample with 20\% normal cell contamination, $30 \%$ cells from subclone A and 50\% cells from subclone B. In grid plots each data point represents average CNs of a genome segment. Different colors represent genome segments with different behaviors in terms of their average CNs across the sample cells. In this grid plot each third of the genome results in a separate grid pattern (blue for subclone B integer CNs $(1,1)$, black for $(0,1)$ or red for $(1,2)$ ) their size determined by the fraction of subclone A cells. The three grids are positioned in a larger grid for which the size is determined by the larger fraction of subclone B cells (green). 
condition 1 includes the point $(1,1)$, the integer $\mathrm{CNs}$ of all other subclones are normal $(1,1)$ in the genome segments of the points for which condition 1 is true. However, condition 1 is seldom truly known for any points (Figure 3a). Therefore, integer $\mathrm{CN}$ estimation in subclonal tumor samples can only be done from noisefree average CNs if the sample has certain properties and under certain assumptions. In Materials and methods we further demonstrate $\mathrm{CN}$ estimation challenges caused by selected subclonal structures through Figure 3, and outline properties and assumptions under which subclonal CNs can be estimated.

\section{Purity versus cell fraction}

$\mathrm{CN}$ alterations in tumor cells appear diluted in average CNs because of the germline (normal) cells in the sample (Figure 1), which are always present. If a sample is known to be clonal, the purity of the sample can be deduced from the density of an observed average $\mathrm{CN}$ grid pattern via Equation 1: the distance between consecutive grid points is equal to the purity. However, when we study tumor samples we usually do not know whether or not that sample is clonal. In this case, as acknowledged by Durinck et al. [2], further, indistinguishable dilution occurs when $\mathrm{CN}$ alterations are present only in part, but not all, of the tumor cells (Figure 2). With or without heterogeneity, the density of a grid pattern in a grid plot holds information about the cell fraction which express $\mathrm{CN}$ alteration throughout some genome segment(s) in which other subclones have constant CNs: the distance between consecutive grid points is equal to that cell fraction (Equation 2). Average CNs do not carry sufficient information to deduce sample purity, although it is sometimes suggested that they do [1,22].

\section{Scaling: where is $(1,1)$ ?}

Summarizing the preceding discussion, cell fractions and integer $\mathrm{CNs}$ can be quantified from unbiased, noise-free average $\mathrm{CNs}$ for some subclones and for some genome segments, if the tumor sample has some fortunate properties and we rely on a set of assumptions. Array CNs are at best proportional to the average CNs in the sample hybridized. Even if they were noise and bias free, array $\mathrm{CNs}$ are insufficient for determination of an identified subclone's integer CNs, its cell fraction and the scaling factor without further information [1,22,23]. The colored points in the simulated grid plot of Figure 4 appear in a regular grid pattern as marked by dotted lines,
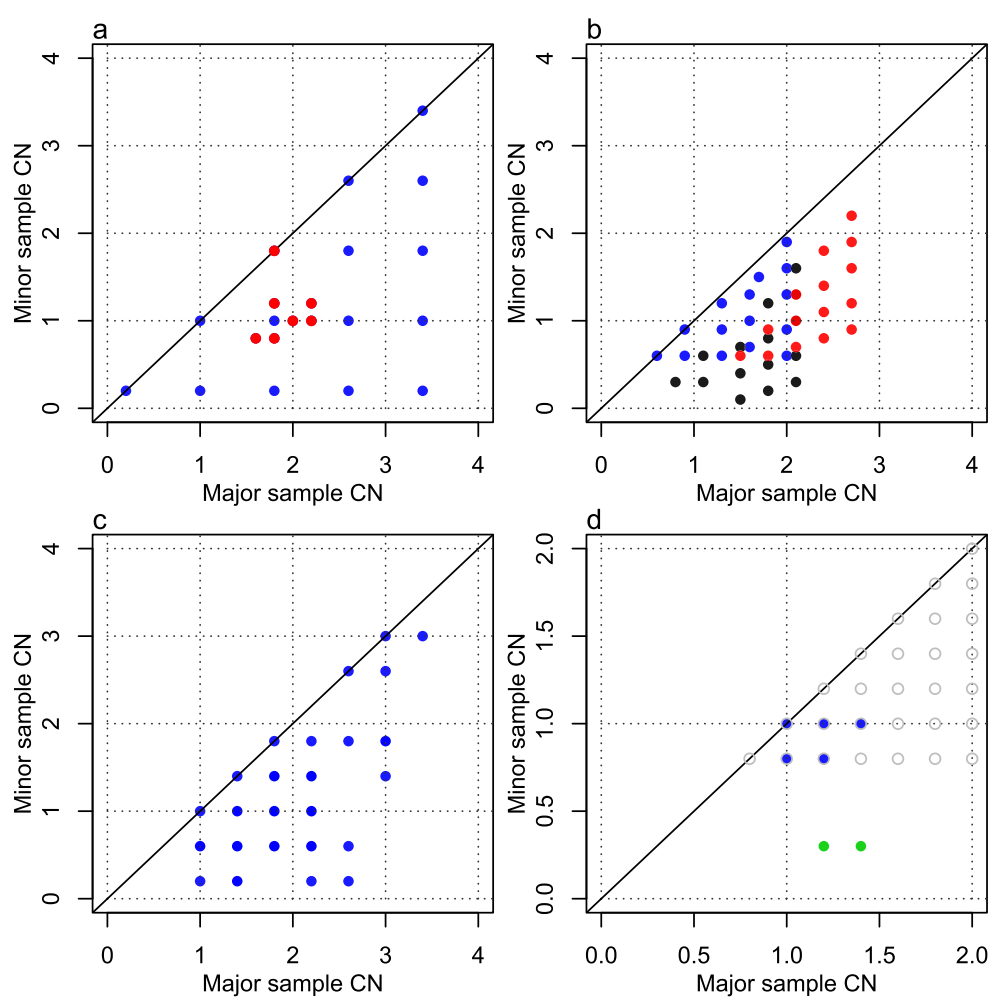

Figure 3 Grid plots of simulated tumor samples with different subclonal architectures. (a) Aneuploid tumor with further subclonality in a small part of the genome. (b) Tumor with three subclones, all with CN alterations. (c) Tumor with two subclones of equal size. (d) Tumor with two subclones of different sizes. Note that each data point in a grid plot represents the average CN across all sample cells. Different colors do not represent different subclones, but highlight specific parts of the genome which we discuss further in Materials and methods. 
but it is unknown which lattice point corresponds to integer copies $(1,1):\left(g_{2}, g_{2}\right),\left(g_{3}, g_{3}\right)$ or $\left(g_{4}, g_{4}\right)$ ? Each of the colored points must have at least 0 minor integer copies. Therefore, the grid pattern suggests that $g_{4}$ is at least 1 , or that $(1,1)$ falls no higher than $\left(g_{4}, g_{4}\right)$. In Materials and methods we explain how the three proposed $(1,1)$ scenarios originate from different integer $\mathrm{CNs}$, cell fractions and scaling factors but result in identical array $\mathrm{CNs}$, or, equivalently, identical total (minor + major) array $\mathrm{CNs}$ and $\mathrm{B}$ allele fractions.

Pounds et al. [23] suggest solving this issue by identification of genomic regions with normal CNs (RAP, reference alignment procedure), which may be possible for some samples but not for all. In the context of heterogeneity, VAFs can sometimes provide sufficient information, and these are used in the software Absolute [1] together with database knowledge about chromosome arm level $\mathrm{CN}$ alterations in common cancer types. Carter et al. [1] and Pounds et al. [23] both stress that manual care with each sample is vital for correct $\mathrm{CN}$ estimation. We examine circumstances under which knowledge of overall sample ploidy, matched normal sample array CNs or VAFs can resolve the scaling issue below.

Ploidy can sometimes help A sample's overall ploidy is the sample's average (minor + major) integer $\mathrm{CN}$ across the genome and across all subclones. In Materials and methods we explain how an independent overall ploidy estimate (for example, from a fluorescence-activated cell sorting (FACS) run) may help us resolve the true position of $(1,1)$. Often, overall ploidy estimates are not given by FACS, but with samples having simple subclonal architecture we may compare subclone-specific ploidies estimated for each potential position of $(1,1)$ (Materials and methods) to the suggested subclone ploidies from FACS, and deduce the true position of $(1,1)$. Figure 5 shows FACS ploidy profiles and segmented SNP array data grid plots for two samples. Sample 11 (Figure 5a,b) has several subclones and integer CNs cannot be located to specific subclones. Sample 29 (Figure 5c,d) has most of its CN alteration in one subclone and the FACS and grid plots combined give clues to the scaling of array CNs.

\section{Paired normal SNP array normalization helps in theory} If array CNs have been normalized towards matched normal tissue SNP array CNs, segments with minor + major array $\mathrm{CNs}$ equal to 1 (red lines in the example samples of Figure 6) and allelic balance (black lines in Figure 6) - that is, segments at the intersection of the two lines - should theoretically correspond to normal integer CNs $(1,1)$. Several CN packages (SOMATICS [24], PICNIC [25], SiDCoN [26], GAP [27] and ASCAT [22]) rely on normalized array $\mathrm{CNs}$ and assume the solution with the minimal possible $\mathrm{CNs}$ that fit their (grid) pattern. 

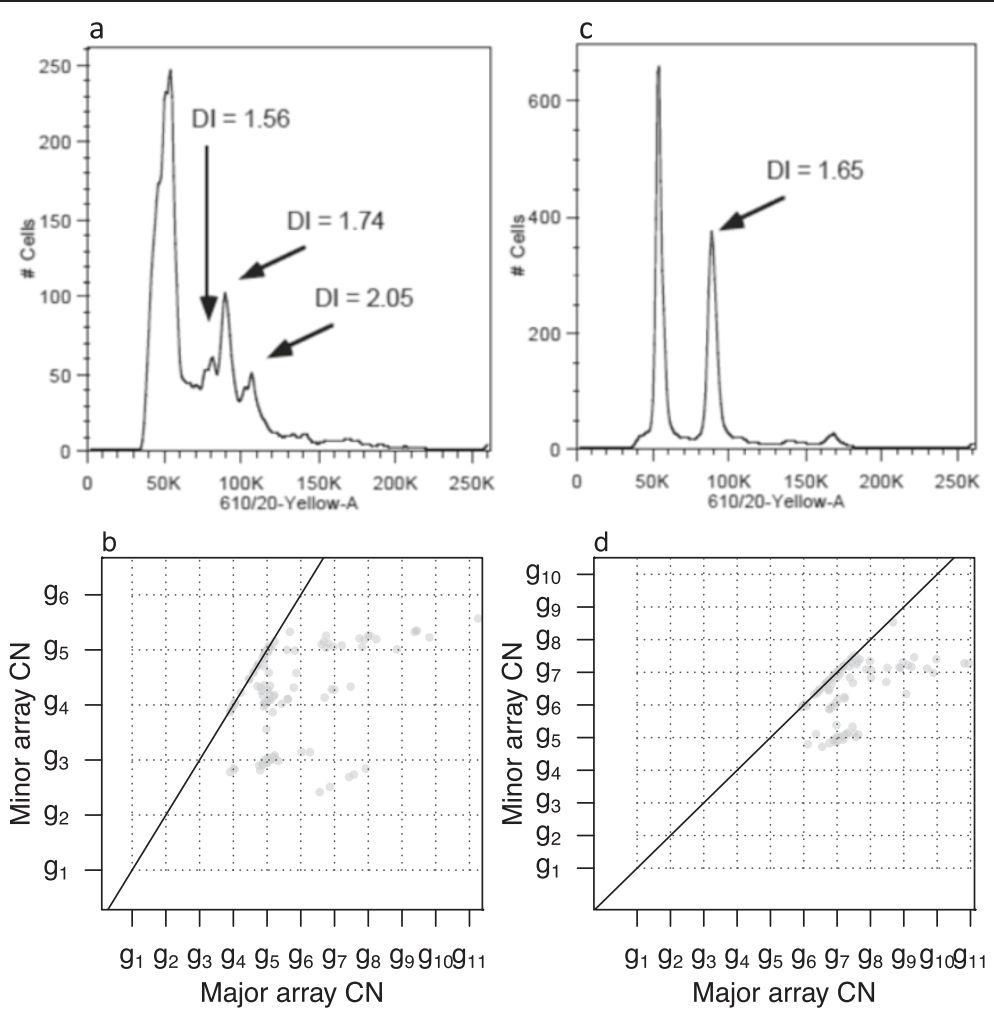

Figure 5 FACS ploidy profiles. FACS ploidy profiles (upper panels, number of cells versus cell ploidy) and grid plots (lower panels) of three actual data samples. Peaks at $50 \mathrm{~K}$ in the FACS profile correspond to diploid cells. (a) Sample 11 has multiple subclones suggested by multiple peaks in the FACS profile. Some peaks comprise approximately the same cell fraction (peak height). (b) A grid pattern appears in the sample 11 grid plot, but since the FACS profile reveals several subclones of the same size, assumption 1 is not reasonable and integer CNs cannot be located to specific subclones: CN alteration which agrees with the lattice points could originate from any of the subclones of the corresponding size. (c) The sample 29 FACS profile suggests that the largest non-diploid subclone has ploidy 3 to 4. (d) The sample 29 grid plot suggests a subclone with ploidy 3.05 if $g_{6}=1$, ploidy 5.05 if $g_{5}=1$, ploidy 7.05 if $g_{4}=1$, and so on. The FACS profile and grid plot thereby together suggest that $g_{6}=1$ for sample 29. DNA Index (DI) is a measurement of ploidy.

ASCAT notes that they go wrong if that assumption is not correct. A look ahead at our actual data grid plots (Figure 6) suggests that this method will not work in general for our samples.

VAFs can sometimes help For samples with a reasonable amount of mutations in 'informative' locations,
VAFs can help deduce the scaling of array CNs if we rely on a set of chosen assumptions. We outline such a framework in Materials and methods through Figure 4.

\section{Estimation of cell fraction, integer CNs and average CNs}

The cell fraction of subclone A and its integer CNs in genome segments that coincide with the subclone's

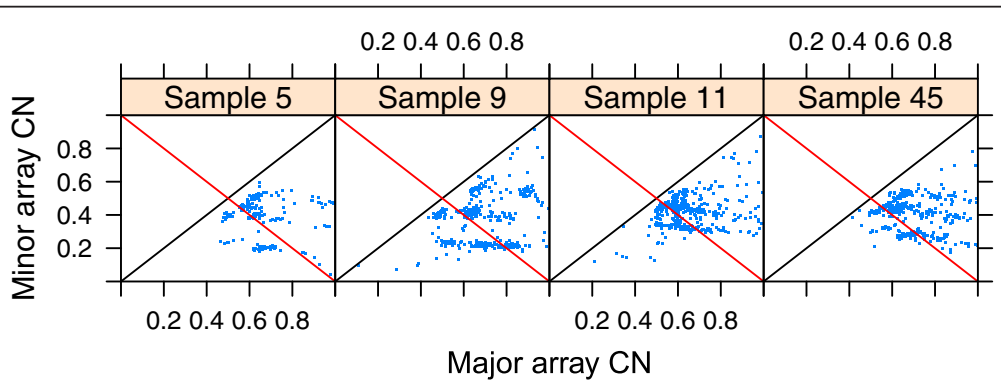

Figure 6 Array CNs normalized towards matched normal tissue SNP array CNs for four actual data samples. Theoretically, points in a cluster around the intersection of allelic balance (black line) and total array CN equal to 1 (red line) should reflect segments with integer CNs $(1,1)$. In practice, the cluster closest to the intersection may reflect $(1,1)$ (samples 5 and 9) but not always (samples 11 and 45 ). 
lattice points (type A segments) can be estimated under the fortunate circumstances described, given the properly scaled array CNs. Let ( $\left.g_{\text {normal }}, g_{\text {normal }}\right)$ be the position of $(1,1)$ and $\Delta$ the distance between two consecutive grid lines. Then we can derive the cell fraction $\alpha=\Delta / g_{\text {normal }}$ of subclone $\mathrm{A}$ and its integer $\mathrm{CNs} c_{1}=\left(a_{1}-g_{\text {normal }}-\Delta\right) / \Delta$ and $c_{2}=\left(a_{2}-g_{\text {normal }}-\Delta\right) / \Delta$, where $\vec{a}_{j}=\left(a_{1}, a_{2}\right)$ are minor and major array CNs of unknown scale. The average CNs can be derived as $e_{1}=a_{1} / g_{\text {normal }}$ and $e_{2}=a_{e} / g_{\text {normal }}$.

\section{B: Clonal or subclonal mutations}

With cell fractions and integer $\mathrm{CNs}$ of one or more subclones resolved and with knowledge of the sample purity, we can assess whether a VAF suggests the corresponding mutation is clonal (present in all tumor cells) or subclonal (not present in all tumor cells) if we rely on previously outlined and further properties and assumptions (see Materials and methods).

The simulated sample of Figure 4 provides an example. Figure $4 \mathrm{c}$ gives the integer $\mathrm{CNs}$ and cell fraction of the sample's main subclone A. The genome segment of the blue grid point (Figure 4a) has no $\mathrm{CN}$ alteration in any cells. The blue column in Figure 4c shows expected VAF levels of heterozygous mutations present only in the cells of the main subclone (thick continuous horizontal line), present only in all other tumor cells (bottom thick dashed line) or present in all cells (top thick dashed horizontal line) of such genome segments. The observed VAF (red cross) coincides with the latter, so the corresponding mutation is estimated to be clonal. The red genome segment (Figure 4a) has CNs $(1,2)$ in the main subclone, and normal CNs in all other cells. The pink column of Figure 4c shows the expected VAF levels given these CNs (thick horizontal lines). The top two thick dashed horizontal lines reflect expected VAF levels of clonal heterozygous mutations present on all its homologue's copies. The mutation on this segment is hence estimated to be clonal too. If it had coincided with one of the lower horizontal lines, we would have estimated it to be subclonal.

The four colored columns of Figure 4c show different expected VAF levels of clonal mutations (top one or two thick, dashed horizontal lines, one or two depending on whether the minor and major integer $\mathrm{CNs}$ are equal or not), and different expected VAF levels of mutations present only in the main subclone A (thick, solid horizontal lines), resulting from different local integer CNs. We also note that other cell fractions of subclone A, together with other integer CNs (Figure 4b,d), would give other expected VAF levels. Two important conclusions follow. First, in order to enable classification of mutations as clonal or subclonal from VAFs with any precision, correct estimation of subclonal integer $\mathrm{CNs}$ and cell fractions is vital. (The procedure will still rely on simplifying assumptions, even for mutations on fortunate segments on grid plot lattice points of an identifiable subclone, and when there is no noise or bias in VAFs or segmented $\mathrm{CN}$ data.) Second, one subclone is associated with a whole set of expected VAF levels, dependent on the subclone's cell fraction and integer CNs, for example, the thick continuous horizontal lines in Figure $4 \mathrm{~b}-\mathrm{d}$. This contrasts with what has sometimes been suggested [8]. We return to this point in the Discussion.

\section{C: Data examples}

In this section we illustrate what we learned in the previous sections through selected analyses of single tumor and matched normal samples from a set of 52 newly diagnosed HER2-positive breast cancer tumors. The patients were all part of a European Union funded project (RESPONSIFY) investigating biomarkers of resistance to trastuzumab plus chemotherapy, which is standard treatment for newly diagnosed breast cancers that have HER2 amplification. Clinical follow-up data are available for each patient through a median time of 5 years, including relapse status. SNP arrays and WES were run as described in Materials and methods. Tumor sample purities (fractions of tumor cells) were estimated by a pathologist. The median purity was $87 \%$ across tumors. For details about SNP array preprocessing and detection of mutations, see Materials and methods.

\section{Bias in BAF can cause skewness in SNP array data}

Our observed array $\mathrm{CN}$ grid plots display skewness (Figure 7), so that segments with the same minor $\mathrm{CN}$ appear in clusters on a sloping rather than a horizontal line, and segments which have the same major $\mathrm{CN}$ appear in clusters on a sloping rather than a vertical line. This phenomenon is particularly pronounced in those of our samples that do not have matched normal sample SNP array data. This is an artifact caused by a systematic bias in our SNP array BAFs which needs to be removed in order to make the $\mathrm{CN}$ estimates comparable to WES VAFs. We do this by grid rotation and describe the BAF bias (see Materials and methods). Unless otherwise stated, we refer to array $\mathrm{CNs}$ as rotated array CNs.

\section{A typical HER2+ grid plot}

Most of our HER2-positive breast cancer sample grid plots are similar to Figure 8, which suggests that they have an aneuploid subclone A in a small fraction of cells (because the regular grid pattern is small). Many of the grid plots also have some short segments with minor array CNs below the most prominent grid pattern, as in Figure 8, which suggests that a larger subclone than the aneuploid one has some LOH. 

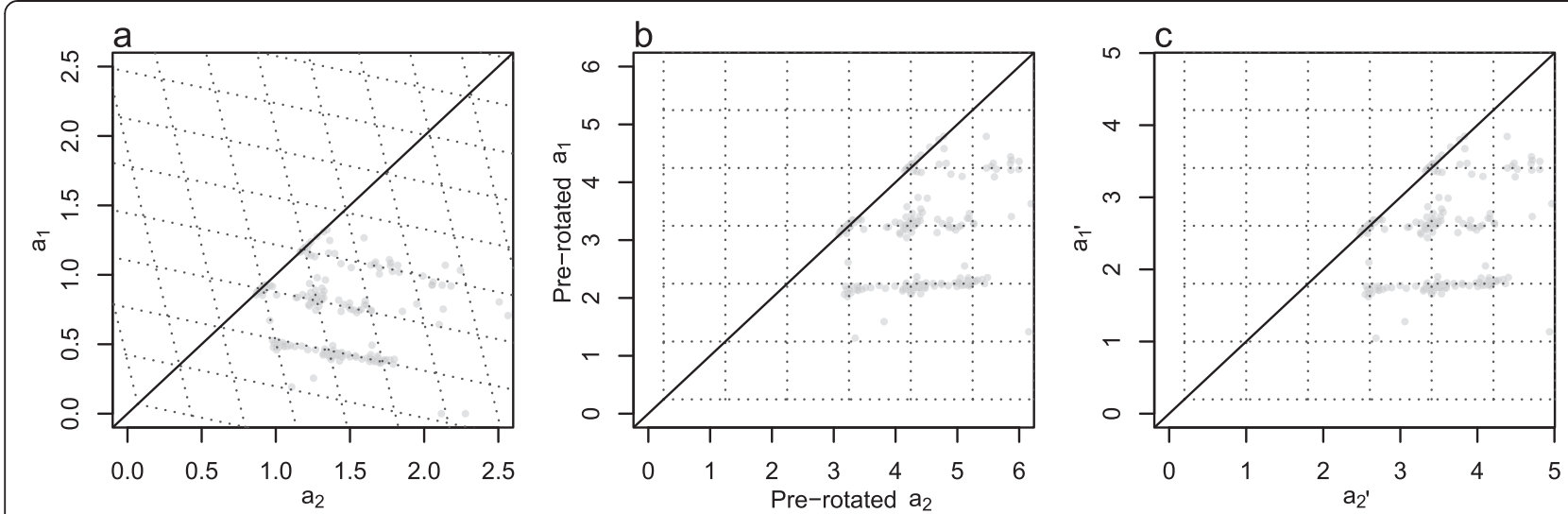

Figure 7 Sample 45 grid plots. For the description of notation see Materials and methods. (a) Original array CNs with skewness. (b) Array CNs after pre-rotation in search of start values (see Materials and methods). (c) Array CNs after completed grid rotation, which removes the skewness. Unless otherwise stated, we refer to array CNs as rotated array CNs and we drop the prime from $\rightarrow a^{\prime}$.

Probabilistic model to separate subclonality from noise and a simple endpoint quantifying ITH

In Figure 8 we identify a regular grid pattern (type A segments, blue), possibly caused by $\mathrm{CN}$ variation in a subclone, say A, of cells. We also spot array CNs that do not follow the grid pattern, in between the regular lattice points (type B segments, pink). In general we see lattice points (type A) as the default location of grid plot points, and it is only if we observe significant evidence to the contrary that we set the type of a segment to $B$ according to the following process.

The classification between type A and B segments is made through the two-dimensional distribution of grid points $\left\{\vec{a}_{i}\right\}$ relative to their closest lattice points $\left\{\vec{e}_{i}\right\}$, in effect overlaying all the lattice points into $\left\{\vec{x}_{i}=\vec{a}_{i}-\vec{e}_{i}\right\}$ (Figure 9a). We fit a two-dimensional $t$-distribution [28] centered at the origin to the $\left\{\vec{x}_{i}\right\}$, with maximum robustness (degrees of freedom $=2$ ) in order to capture the variation of observations in the dense central cluster (which may truly have $\mathrm{CN}$ alteration in subclone A only) but not that of the many outliers (which may not originate from $\mathrm{CN}$ alteration in subclone $\mathrm{A}$ ). The estimated covariance matrix $Q$ is used to calculate a segment length-weighted squared Mahalanobis distance $M_{i}^{2}=\vec{x}_{i}^{T}$ $\left(Q / w_{i}\right)^{-1} \overrightarrow{\mathbf{x}}_{i}$ for each segment $i$, which should follow an exponential distribution with scale parameter $1 / 2$ for segments within the dense centre cluster. We choose a cutoff $m$ where the linearity in the exponential $q q$-plot starts to

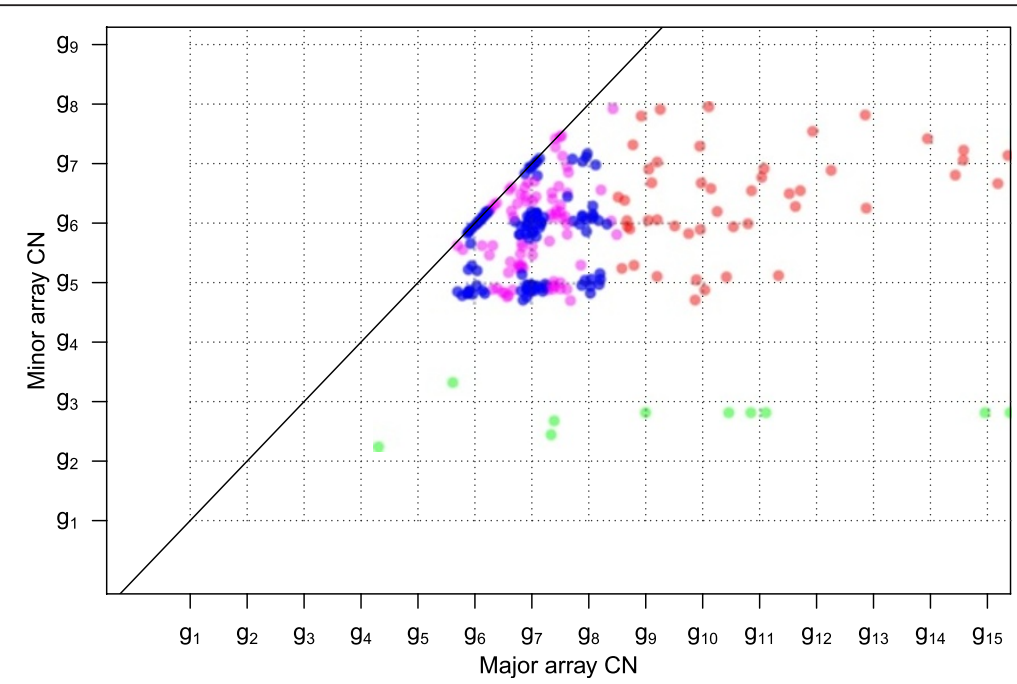

Figure 8 Sample $\mathbf{5}$ grid plot. The small, regular grid suggests that a low fraction of the sample cells form an aneuploid subclone (A). The segments with minor array CNs far below the regular grid may be caused by LOH in a different, larger subclone. Segments are classified into types A (blue, regular grid pattern), B (pink, breaking regular grid pattern), C (green, lower array CNs than the most evident grid pattern) and D (red, high enough array CNs for regular grid patterns not to appear). 

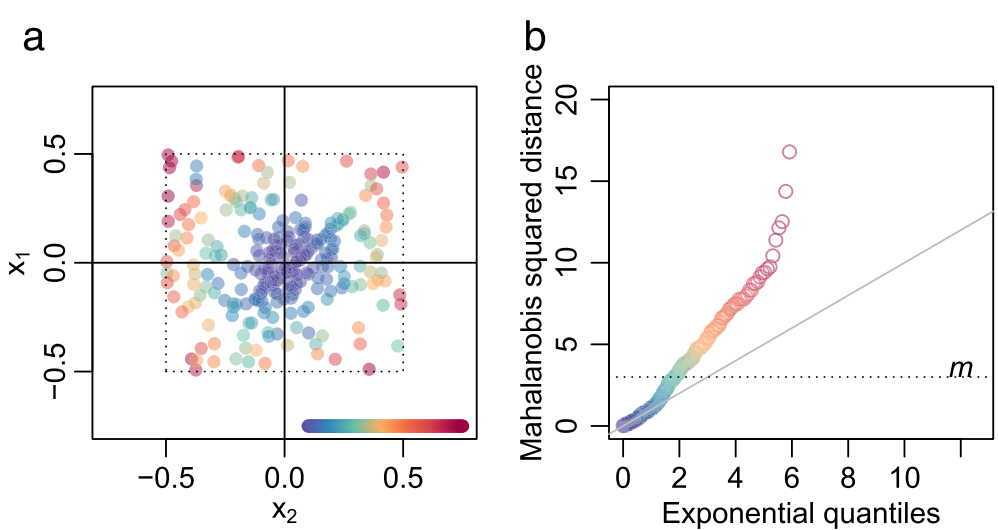

Figure 9 Noise or heterogeneity in sample 5. (a) Positions of array CNs relative to their closest grid plot lattice points, with coloring by statistical distance. Note that positions have been scaled to fit the lattice point and its closest segments within \pm 0.5 . (b) Exponential(1/2) 99 -plot with the same coloring as (a). Points above the horizontal cutoff $(m)$ indicate segments with array CNs significantly different from the lattice points (type B segments).

fail (Figure 9b; commonly conservatively chosen to $m=3$ ), and classify segments as type $\mathrm{A}$ if $M_{i}^{2} \leq m$ and type $\mathrm{B}$ otherwise. The segment length weight $w_{i}=1-e^{-l_{i} / 500000}$, where $l_{i}$ is the length of segment $i$, downweights $M_{i}^{2}$ values of short $(<1 \mathrm{Mb})$ segments, since we think their deviance from the origin may be due to noise in the array $\mathrm{CNs}$ of such small segments, rather than to a true patternbreaking deviance in CNs.

The fraction of the genome covered by type $B$ segments out of that covered by type A and B segments is a simple measure of the amount of ITH in a sample. This endpoint estimates the fraction of the genome in which the sample has $\mathrm{CN}$ alteration in other subclones than the main subclone A (possibly in addition to $\mathrm{CN}$ alteration in A). It has proved useful for prediction of relapse in the RESPONSIFY samples. Details will be published separately.

\section{Scaling: resolving location of $(1,1)$ with help of VAFs and purity}

We estimate the scaling of a sample's array CNs by the scenario that best fits VAFs estimated from WES data for mutations in segments classified to be of type A with respect to the sample's most evident subclone A. By our assumptions, these segments have $\mathrm{CN}$ variation only in the subclone A cells. Out of the 52 RESPONSIFY samples the scaling was resolved in this manner for 48 samples.

In Figure 10 we display a typical example rather than a perfect one (as, for example, that of Figure 11 below). Each panel shows the five expected VAF levels (y-axis, horizontal lines with different colors) for each type A $\mathrm{CN}$ segment ( $\mathrm{x}$-axis, ordered by decreasing expected VAF if present only on non-A cells and by increasing minor + major array $\mathrm{CN}$ ) for one potential position of $(1,1)$ of the sample introduced in Figure 8 under assumptions 3 to 6 in Materials and methods. The observed mutation VAFs of type A segments are shown as red crosses. Each panel also gives SS, the sum of squared distances to each VAF's nearest expected VAF level. The figure suggests that $g_{3}=1$ or $g_{6}=1$, since in these panels the observed VAFs are, on average, closer to their expected levels (they have lower SS than the other panels). Note that we do not expect all mutations in type A segments to follow our assumptions and fit one of the expected levels, but we assume that most mutations do, in order to resolve the scaling of the array CNs.

To further differentiate between the two suggested scenarios we transform these panels' $y$-levels to show subclone A integer $\mathrm{CN}$ estimates under these scenarios (Figure 12), also showing the segments by their genome position. Now, the (dark) green and blue horizontal lines are the minor and major integer $\mathrm{CN}$ estimates of subclone A for segments that have $\mathrm{CN}$ alteration only in subclone A. The red crosses' y-levels show the mutation multiplicities (calculated under the assumption that they sit on $\mathrm{A}: \frac{D}{\alpha} V A F$ ), which equal an integer $\mathrm{CN}$ estimate (green or blue horizontal line) if the mutation VAF equals the corresponding expected VAF level. The black line shows the expected y-level of the multiplicity for mutations on the single copy of all tumor cells not in A, which is equal across all segments. The light green and light blue horizontal lines show the expected y-levels of clonal mutation multiplicities. We see that if $g_{3}=1$, subclone A has no single allele integer $\mathrm{CN}$ below 3, a rough calculation (see Materials and methods) suggests the sample has overall ploidy above 4, and all mutations seem to sit only on the assumed diploid cells (black horizontal line), not in subclone A. If $g_{6}=1$, subclone A 

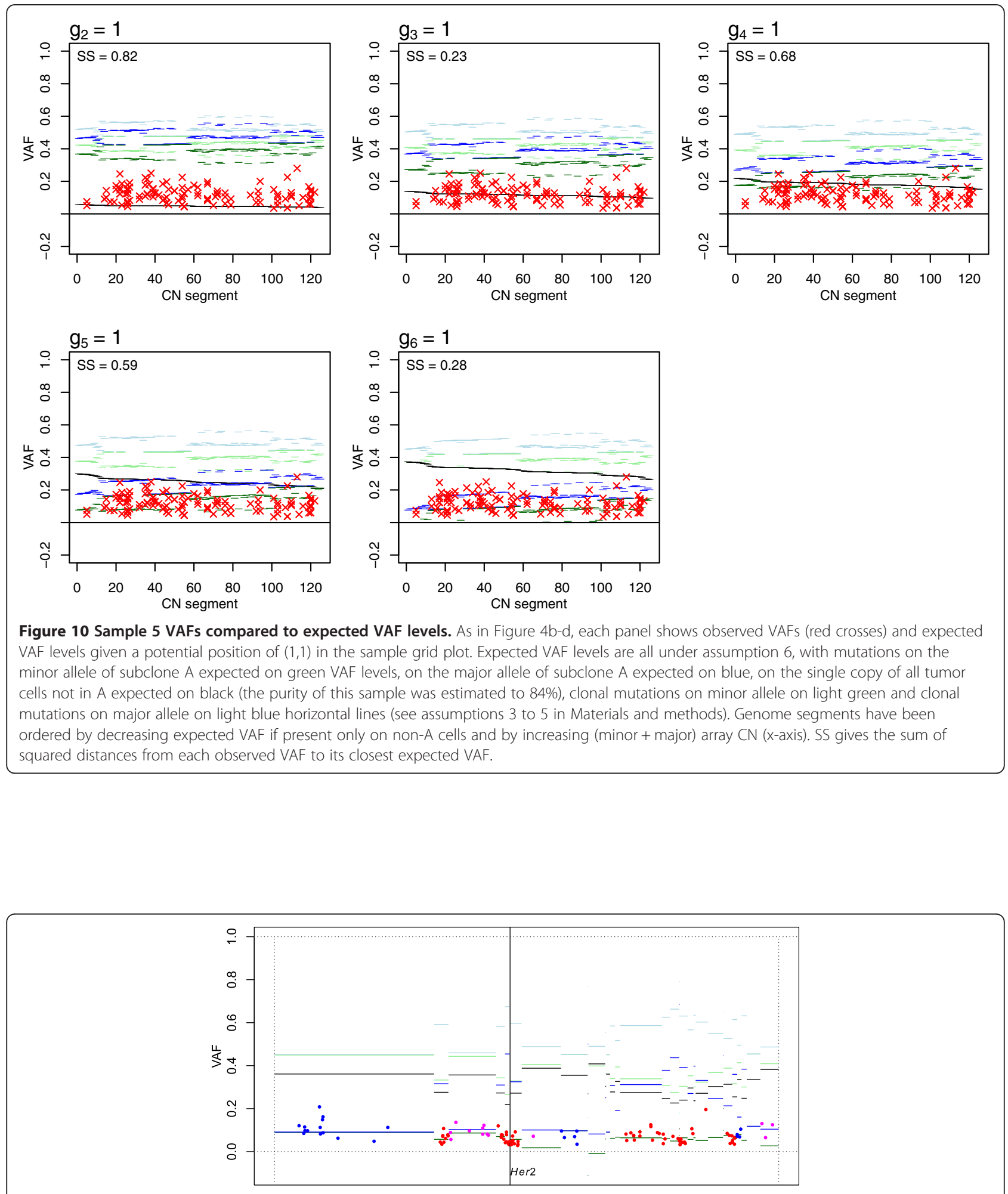

Position along chromosome 17

Figure 11 Sample 16 VAFs fit expected VAFs along chromosome 17. Sample 16 VAFs (points) and expected VAF levels (horizontal lines; Figure 10) along chromosome 17 given subclone A CN estimates and its sample cell fraction $a$. This sample has 1,232 detected mutations, which is many more than the median 156 of the 52 RESPONSIFY samples. We see an almost perfect fit of the observed VAFs to those expected. For color coding of VAFs, see text. This figure also shows how the mutation rate differs across the chromosome, a different type of heterogeneity studied by Lawrence et al. [29]. The purity of this sample is unknown and is imputed to $90 \%$. 


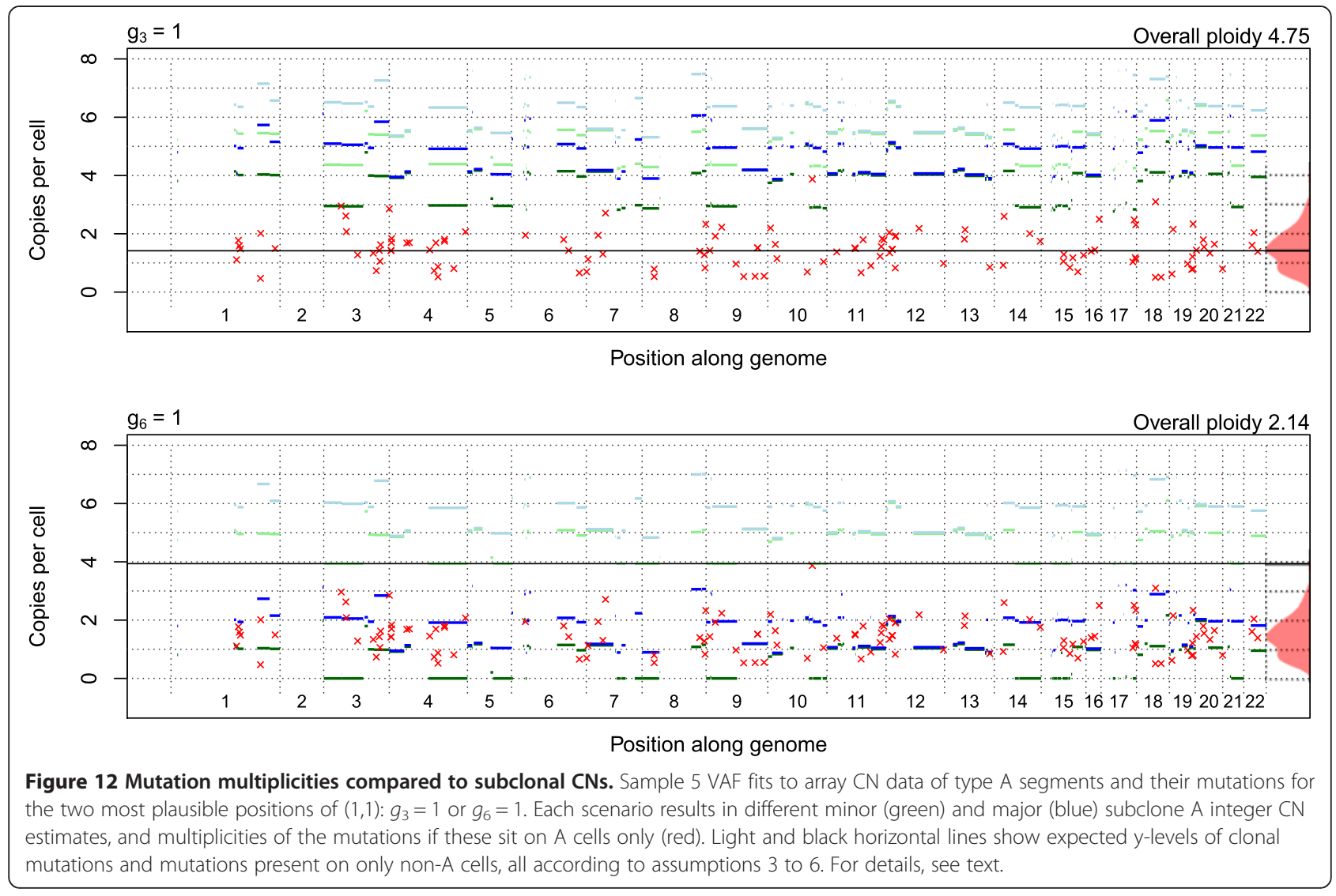

has single allele integer $\mathrm{CNs}$ from 0 and above, the rough overall ploidy estimate is just over 2 and most mutations seem to sit on the subclone A cells. The majority of our samples end with a similar choice. The $g_{6}=1$ scenario sounds more reasonable and therefore we choose to proceed with that. When in doubt we choose the conservative scenario with the smallest integer $\mathrm{CNs}$ and smallest size $\alpha$ of subclone A.

See Materials and methods for our suggested estimation of subclonal architecture, cell fractions and integer CNs.

\section{Clonal or subclonal mutations}

Our classification of mutations as clonal or subclonal is based on the methods outlined for simulated data. To acknowledge the uncertainty of real VAFs we run a set of non-inferiority, inferiority and equality tests for each VAF based on its binomial two-sided 90\% confidence interval (CI) from the sequencing number of variant versus reference reads. For details, see Materials and methods.

\section{Clonal or subclonal $\mathrm{CN}$ alterations}

The vertical continuous line in Figure 11 denotes the position of the HER2 (ERBB2) gene. Our samples have been diagnosed as HER2-enriched, and they do have a high, type $\mathrm{D}$, major array $\mathrm{CN}$ at this position. Unfortunately no samples have VAFs that match the major homologue, so it is not possible to assign the clonality status or subclonal origin of HER2 enrichment. The number of detected mutations in the 52 RESPONSIFY samples varies from 1 to 1,232 (median 156.5), and only a handful of samples have enough mutations (say >900) to enable assessment of subclonal origin of $\mathrm{CN}$ alteration on a large scale.

\section{Discussion}

The aim of this paper is to highlight challenges in $\mathrm{CN}$ estimation that influence mutation classification but are infrequently acknowledged in the literature as well as propose solutions that may aid in the quantification of ITH in tumor samples that have high levels of $\mathrm{CN}$ alteration. We have demonstrated how even with no noise or bias, integer $\mathrm{CNs}$ of tumor samples with ITH can only be estimated from segmented $\mathrm{CN}$ data in samples with a simple clonal architecture, given further information from, for example, WES VAFs or FACS images, and under a series of assumptions. Even with such samples, integer CNs can only be deduced for some subclones and only across a subset of the genome.

Classification of mutations as clonal or subclonal further requires knowledge of the sample purity, which 
cannot be deduced from segmented $\mathrm{CN}$ data. The classification relies on comparing observed VAFs to expected VAF levels given purity, subclonal cell fractions and local $\mathrm{CNs}$. Therefore, the assumptions made in the $\mathrm{CN}$ estimation procedure will have a large influence on how mutations are classified, and on how the results can be interpreted.

We have also suggested a simple ITH endpoint for tumor samples with a high level of $\mathrm{CN}$ alterations based on segmented $\mathrm{CN}$ data alone and which does not require knowledge of subclonal cell fractions or integer CNs.

\section{Estimate average $\mathrm{CNs}$ from sequencing}

We have used SNP arrays to derive segmented $\mathrm{CN}$ data for the RESPONSIFY samples. Alternatively, sequencing depths could be used [12-17], which has the advantage that it works well on formalin-fixed paraffin-embedded tissue, whereas SNP arrays usually require frozen tissue, which is less practical to validate. The expected (true) average $\mathrm{CN}$ patterns of tumor samples with heterogeneity are the same whether average CNs are estimated from SNP arrays or sequencing data. The challenges presented hence apply either way: there is ambiguity between purity and heterogeneity, there are difficulties deducing subclonal structures and assigning a subclonal origin to a segment with $\mathrm{CN}$ alteration, and the scaling of array CNs or SNP position sequencing depths relative to average CNs is unknown. Both sequencing and SNP array data may suffer from bias which needs attention before estimation of average CNs, although the types of bias are different. There may be BAF bias in SNP array data and GC bias in sequencing depths. Standardizing tumor sample sequencing depths to matched normal sample sequencing depths comes with challenges that are different from those of standardizing tumor sample array $\mathrm{CNs}$ to matched normal sample array $\mathrm{CNs}$. We generally seek more evidence that the results after different steps of analysis look plausible than is typically presented in a literature dominated by model-based inferences. We find that just as important as detailed model descriptions. As for $\mathrm{CN}$ determination, a study of the two-dimensional grid plots (applicable equally well to SNP array CNs and sequencing depths) of average $\mathrm{CN}$ estimates can help reveal bias and give clues to sample architecture.

\section{Whole genome sequencing versus whole exome sequencing}

WGS identifies many more mutations than WES (which can only find mutations in gene exons), but is comparatively more expensive. More mutations help in assigning $\mathrm{CN}$ alterations to identified subclones, and resolving the scaling of segmented $\mathrm{CN}$ data in relation to average CNs. Therefore, WGS is generally a benefit for assessment of integer CNs, clonality of mutations or phylogenetic trees (see below) in subclonal tumor samples.

\section{Phylogenetic trees}

Given a set of identified subclones in a sample, trees can be inferred by assigning mutations to subclones and checking whether mutations close in genomic location but assigned to different subclones tend to co-appear or never co-appear on the same fragment. Co-appearance indicates that one of the subclones is in turn a subclone of the other, and no co-appearance indicates that the subclones belong to independent branches of the tree. Given that WGS identifies more mutations than WES, WGS is again a benefit. Since the majority of our samples have too complicated subclonal structures for more than one or two subclones to be identified in detail, and relatively few mutations identified by WES, detailed phylogenetic trees are not generally within reach. The number of identified mutations in the 52 RESPONSIFY samples ranges from 1 to 1,232 (median 156.5; samples were selected so that they had at least one identified mutation). The WES average coverage of the samples ranges from 25 to 179, with median 108.

\section{Clustering of cancer cell fractions}

It has been suggested $[8,9,12-17]$ that, with WGS, subclones can be identified via groups of mutations present in similar fractions of cancer cells. On this topic we would first like to stress that clustering of a sample's VAFs is something different from clustering of the sample's cancer cell fractions. The former may cluster because of aneuploidy in the sample, even if the sample has no heterogeneity: a sample with aneuploidy has several expected VAF levels (like the thick continuous horizontal lines in Figure 4c), so each subclone corresponds to several VAF clusters. Also, different subclones may have overlapping expected VAF levels.

To the best of our knowledge, Papaemmanuil et al. [9] do not take local $\mathrm{CNs}$ into account when classifying mutations as clonal or subclonal. They assume that the mutations with the highest VAFs are clonal, and classify mutations as subclonal if their CIs do not overlap with those of the 'clonal' mutations. As seen in Figure 11, expected clonal VAF levels (light green and light blue horizontal lines) may be very close to expected subclonal VAF levels (black horizontal lines). Therefore, we do not generally recommend classification of mutations by comparing a sample's VAFs within themselves with no reference to local integer CNs.

Nik-Zainal et al. [8] estimate the cancer cell fraction (ccf) of each mutation by what we call the 'multiplicity' of the mutation given integer $\mathrm{CN}$ estimates in the most evident identified subclone. For RESPONSIFY sample 5, this is exactly the y-levels of mutations in Figure 12. 
More precisely, Nik-Zainal et al. [8] estimate ccfs as the minimum of the multiplicity and 1 , and look for clusters among the mutations with $\mathrm{ccf}<1$. We see no clusters among the y-levels of mutations below the dotted horizontal line of 1 in Figure 12, but perhaps we have too few mutations of type A detected from the WES data. We note that such clusters would only reveal very small subclones with low integer $\mathrm{CNs}$ and daughter subclones of the most evident identified subclone. We also note that with this ccf estimator, mutations of such a small subclone will get different ccf estimates if they sit on segments with different integer CNs in the most evident identified subclone, so several clusters may arise from the same subclone. Nevertheless, this method may help screening for long subclonal $\mathrm{CN}$ alterations to be verified by phasing of SNPs and mutations on the same sequencing reads, which is what Nik-Zainal et al. [8] do.

The PyClone algorithm [14] clusters mutations on the basis of their VAFs corrected for local $\mathrm{CN}$, termed the 'cellular prevalence.' To do so, at each mutation the algorithm splits the cells in the sample into the 'normal population', the 'reference population', consisting of all cancer cells which do not contain the mutation, and the 'variant population', consisting of all cancer cells with the mutation. It makes a 'key assumption' that all cells within their three populations have the same genotype. We have applied the algorithm to the six samples discussed and made available in this paper. It produces an estimate of the number of subclones in a sample, and assigns mutations to subclones. The results are, in part, consistent with, but also complementary to, ours, bearing in mind that we do not attempt to estimate the number of subclones in a sample. For example, sample 16 depicted in Figure 11 has 1,232 somatic mutations, and PyClone infers 6 clusters, assigning over 900 to one and over 250 to a second. In data not shown, we inferred that $\mathrm{CN}$ alterations in a main aneuploid subclone only (segments of type A) comprised 90\% of the genome and held 839 of the mutations (no others could be assigned to a specific subclone), while we found $8 \%$ of the genome to be segments of type $B$. This is a fair degree of consistency between rather different approaches to the same problem. On the other hand, sample 5 had its 199 mutations put into just 3 clusters by PyClone, but as can be seen from Figures 8, 10 and 12 it has a considerable amount of subclonality, and we see evidence of more than 3 subclones. Most of our samples are like sample 5 in being highly heterogeneous, and it seems likely that the differences between PyClone's results and ours stem from a failure of their 'key assumption', in that we have different $\mathrm{CNs}$ between different subclones. This point is highlighted in [16], where it is noted that clonal inference using $\mathrm{CN}$ aberrations and $\mathrm{B}$-allele frequencies need not be the same as that using somatic aberrations. Our approach and that of PyClone are different ways of integrating these two data types, while the integrative analysis of [16] is perhaps better than both if one has WGS data. Their method is not available to us as we do not have such data.

$\mathrm{CN}$ estimation and mutation classification in the literature Durinck et al. [2] identify $\mathrm{CN}$ neutral LOH regions within one tumor subclone and classify mutations as homozygous or heterozygous within the subclone. This aim is slightly different to ours, but the paper deserves a mention because it acknowledges that an identified $\mathrm{CN}$ pattern reflects $\mathrm{CN}$ alteration in a subclone rather than in all tumor cells.

The software Absolute [1] deduces integer $\mathrm{CNs}$ in pooled minor and major array $\mathrm{CN}$ histograms. The BAF bias of the RESPONSIFY array CNs cannot be spotted with onedimensional histograms rather than two-dimensional grid plots, and in Materials and methods we demonstrate how Absolute therefore does not work with our samples. But given data without bias, Absolute estimates integer CNs under the assumptions that (i) only one pattern of equally interspaced peaks can occur, and (ii) the pattern reflects the clonal CNs of all tumor cells in the sample. With the theoretically expected $\mathrm{CN}$ patterns of Figures 2 and 3 as background we suggest this approach may be useful for samples with most $\mathrm{CN}$ alteration taking place in most of the tumor cells, and only small subclones (accounting for up to say $10 \%$ of the sample cells) expressing further $\mathrm{CN}$ alteration.

To deduce the scaling of the array CNs, Absolute suggests the scenario for which the majority of (all the sample's) VAFs fit presence on one copy of one homologue of the large subclone. We acknowledge that this is different from our suggested scenario with VAFs (from the genome segments with $\mathrm{CN}$ alteration in the pronounced subclone) fitting presence on all copies of one homologue.

Nik-Zainal et al. [8] estimates integer CNs and sample purity with ASCAT [22], and thereby assumes the minimal CNs fitting array CNs (ignoring the unknown scaling) as well as assumptions (i) and (ii) above, as Absolute does. Again we suggest this approach may be useful for samples with most $\mathrm{CN}$ alteration taking place in most of the tumor cells, and only small subclones (accounting for up to say $10 \%$ of the sample cells) with other $\mathrm{CN}$ alteration. Nik-Zainal et al. [8] further refine the precise integer CN estimates with help of WGS depths at SNP positions. This may or may not eliminate any BAF bias in average $\mathrm{CN}$ estimates; a reader of the paper cannot deduce which. ASCAT fails with most of the RESPONSIFY samples, which are highly aneuploid and subclonal.

The methods also differ in their interpretation of mutations as clonal or subclonal. In simple terms we call a mutation clonal if its VAF (is larger than or) fits presence on 
all copies of one homologue in subclone A plus on one copy of one homologue in the rest of the tumor cells (a fraction of cells determined via the pathologist purity estimate). Mutations with significantly smaller VAFs we call subclonal. Absolute calls a mutation clonal if the VAF gives a high likelihood of its presence on at least one copy of a homologue of the (large) subclone. Mutations with a high likelihood of presence in less than one copy are called subclonal. Nik-Zainal et al. [8] similarly call a mutation clonal if it seems present in at least one copy of one homologue of the (large) subclone, except in segments with further subclonality (type B segments) where they require more. The methods will clearly classify mutations differently. Our method of calling clonal mutations is conservative, and will only find a few such mutations per sample (sometimes none, in particular since ambiguous mutations are not classified). The other three methods $[1,8,9]$ are conservative with calling subclonal mutations and will only call those that are present in a small fraction of cells. To our knowledge there is no clear answer to which of these interpretations is more appropriate biologically.

\section{Conclusions}

We have demonstrated that even with no noise or bias, integer CNs of tumor samples with ITH can only be estimated from SNP array data in samples with a simple clonal architecture, given further information from, for example, WES VAFs or FACS ploidy profiles, and only under a series of assumptions. Even with such samples, integer $\mathrm{CNs}$ can only be deduced for some subclones and only across a subset of the genome.

Estimation of local subclonal CNs has implications for the classification of mutations as clonal or subclonal. The classification also requires knowledge of the sample purity, which cannot be deduced from segmented $\mathrm{CN}$ data. The literature on this topic is divergent in assumptions and data analysis methods, with interpretational differences as a result. The insights demonstrated in this study impact research in heterogeneity and tumor evolution, with our emphasis being not only on data analysis methodology but also on the goals, design and interpretation of such studies.

We would like to stress the importance of illustrative figures to reveal bias and verify model assumptions in ITH studies. We think such evidence of performance is just as important as descriptions of analysis models in papers. As for $\mathrm{CN}$ determination, a study of two-dimensional grid plots of average $\mathrm{CN}$ estimates can help reveal biases and give clues to sample architecture.

\section{Materials and methods}

This section provide further details and demonstrations of the points made in the main text.

\section{CN estimation challenges caused by selected subclonal structures}

We aim to outline a set of assumptions under which subclonal cell fractions and integer $\mathrm{CNs}$ can be estimated from average $\mathrm{CNs}$ for some tumor samples. Let us first demonstrate some selected subclonal architectures with help from Figure 3.

The clonal, aneuploid tumor of Figure 1 would have average $\mathrm{CNs}$ as shown in blue in Figure 3a. We simulate a small subclone emerging from the tumor, so that part of a segment which originally had integer CNs $(1,2)$ now splits up into small segments with different amounts of $\mathrm{CN}$ alteration relative to the original, main subclone. Figure 3a shows the resulting grid plot, in which the affected small segments have been colored red. We note that small subclones with additional $\mathrm{CN}$ variation to that of a main subclone will cause average CNs between (and sometimes even on top of) the main subclone lattice points.

Next, imagine a subclonal tumor with 90\% purity, which has two subclones as in Figure 2 plus $\gamma=10 \%$ cells forming another subclone $\mathrm{C}$ with integer $\mathrm{CNs}$ from 0 to 4 , varying independently of the other subclonal integer $\mathrm{CNs}$. Figure $3 \mathrm{~b}$ shows simulated average $\mathrm{CNs}$ of such a tumor sample, where segments from each third of the genome has been colored differently. Presented with such a grid plot, the underlying subclonal architecture is not easily detected. Even if we were told the number of subclones (three), each average $\mathrm{CN}$ is a combination of three subclonal integer $\mathrm{CNs}$, so integer $\mathrm{CNs}$ for individual subclones could not be estimated from average $\mathrm{CNs}$ alone. We note that the pattern of average CNs quickly gets out of hand as subclonality increases, and that average $\mathrm{CNs}$ between regular lattice points may not be caused only by small subclones that deviate from a main subclone (Figure 3a), but also by small subclones with integer CNs independent of those in a main subclone.

Even with only two subclones many samples cannot be resolved from average CNs. Figure $3 \mathrm{c}$ is a grid plot from a simulated tumor sample with two subclones of the same size, which have independently sampled integer $\mathrm{CNs}$ from 0 to 4 . We note that even though one regular grid pattern can be identified in the grid plot, it is not necessarily caused by just one subclone.

A further difficulty is that in reality not all integer $\mathrm{CN}$ combinations will occur, and in particular not in combination with each integer $\mathrm{CN}$ in other subclones. Figure $3 \mathrm{~d}$ shows the grid plot of a simulated sample with two subclones. Two separate regions of the plot show equally spaced grid points (blue and green). The blue points reflect segments with different integer $\mathrm{CNs}$ in the smaller of the two subclones, and $(1,1)$ copies in the larger one. The possible lattice points on which such grid points can fall 
have been circled. The green points reflect segments with different integer CNs in the smaller subclone and $(0,1)$ in the larger one.

\section{Properties and assumptions under which subclonal CNs can be estimated}

In this section we describe some sample properties and assumptions under which conditions 1 and 2 hold so that cell fractions and integer $\mathrm{CNs}$ of subclones can be estimated. Imagine a tumor sample for which the following holds.

Property 1: The grid plot has a regular vertical/horizontal grid made up by at least two points. This indicates that there is $\mathrm{CN}$ alteration in a subclone or in all tumor cells throughout some genome segments where no other subclones have $\mathrm{CN}$ alteration. It may also result from the combined effect of $\mathrm{CN}$ alteration in two or more subclones. In order to proceed, we must simply assume (Assumption 1 below) that is not the case.

For example, the blue grid points of Figure 3d satisfy property 1 . Under the following assumption, condition 1 holds so we can correctly identify a subclone (say A) in the tumor sample by its cell fraction.

Assumption 1: The regular spacing between the grid points of property 1 is caused by consecutive integer $\mathrm{CNs}$ in subclone A.

We now consider

Property 2: The point $(1,1)$ is part of the grid pattern suggested by property 1 , even if there are no actual points at $(1,1)$.

and

Assumption 2: All grid points that fall on a lattice point of subclone A (circled in Figure 3d), have normal integer $\mathrm{CNs}$ in all other subclones than $\mathrm{A}$.

This assumption means that no grid points at the lattice points are due to $\mathrm{CN}$ variation in other subclones, like the top red point in Figure 3a, or points of a second subclone with identical size to A as in Figure 3c.

If in addition to assumptions 1 and 2, we have property 2 holding, then condition 2 is satisfied, and we can estimate integer $\mathrm{CNs}$ of subclone $\mathrm{A}$ in the genome segments which fall at lattice points of subclone A. We will call these segments type A segments with respect to subclone A.

Further subclones may be identified using the same strategy. The point $(1,1)$ will be part of the lattice points for each grid caused by $\mathrm{CN}$ alteration in one subclone when the integer $\mathrm{CNs}$ of the other subclones are normal. Therefore, $(1,1)$ may be regarded as an observed grid point in search of points fulfilling property 1 , even if there is no observed point there. With real data, the position of $(1,1)$ will not be identified until a first subclone like $\mathrm{A}$ is found, so only subsequent subclone identifications can make use of it.
Even other subclones may be quantified under additional assumptions, as exemplified next.

\section{Example of further subclonal cell fraction and integer $\mathrm{CN}$ estimation}

Imagine a tumor sample with an identified subclone A according to properties 1 and 2 and assumptions 1 and 2 , and with

Property 3: The grid plot has at least one point below the lattice points of subclone A. (This indicates another subclone, with a larger cell fraction than A.)

For an example, see the green grid points of Figure 3d. Under the following assumption (which could be varied in different ways), condition 1 holds, so we can correctly identify a subclone (say C) in the tumor sample by its cell fraction.

Assumption 3: The horizontal distance between $(1,1)$ and the average minor average $\mathrm{CN}$ of points below the lattice points of subclone $\mathrm{A}$ corresponds to a difference of integer $\mathrm{CNs}$ in subclone $\mathrm{C}$ of one.

We call segments with grid points falling below the lattice points of subclone $\mathrm{A}$ type $\mathrm{C}$ segments. If we further assume

Assumption 4: All type C segments have integer CNs $(0,1)$ in subclone $\mathrm{C}$.

then we could continue to deduce integer $\mathrm{CNs}$ in subclone A for those type $\mathrm{C}$ segments with grid points on a new set of lattice points, based on assumptions parallel to assumption 2 above.

\section{Identical array CNs can originate from different integer CNs}

Given the unbiased, noise-free array CNs of Figure 4, it is unknown which of the lattice points $\left(g_{2}, g_{2}\right),\left(g_{3}, g_{3}\right)$ or $\left(g_{4}, g_{4}\right)$ corresponds to $(1,1)$ integer copies. The scenarios 2, 3 and 4 involve different fractions $\alpha$ of cells displaying the colored grid point $\mathrm{CN}$ alterations, different sets of integer $\mathrm{CNs}$, and different scaling factors between array $\mathrm{CNs}$ and average CNs. The following algebra shows how two consecutive scenarios (2 and 3 ) can result in identical total (that is, minor + major) array $\mathrm{CNs}$ (TCNs) and BAFs, and hence identical array CNs.

For scenario 3 , let $c_{13}$ and $c_{23}$ denote the integer CNs in the aneuploid fraction $\alpha_{3}$ of cells of an arbitrary genome segment. Scenario 3 implies $\alpha_{3}=\left(g_{3}-g_{2}\right) / g_{3}$ (Equation 2) and a scale factor $f_{3}=g_{3}$ relating array CNs to average $\mathrm{CNs}$. Hence the segment has:

$$
\begin{aligned}
& \operatorname{TCN}_{3}=\left\{\alpha_{3}\left(c_{13}+c_{23}\right)+2\left(1-\alpha_{3}\right)\right\} g_{3} \\
& \text { BAF }_{3}^{\text {upper }}=\frac{\alpha_{3} c_{23}+\left(1-\alpha_{3}\right)}{\alpha_{3}\left(c_{13}+c_{23}\right)+2\left(1-\alpha_{3}\right)} .
\end{aligned}
$$

Next consider scenario 2, for which $\alpha_{2}=\left(g_{2}-g_{1}\right) / g_{2}, f_{2}=g_{2}$ and its integer CNs would be $c_{13}+1$ and $c_{23}+1$ for the same 
segment. We note that $\left(g_{2}-g_{1}\right)=\left(g_{3}-g_{2}\right)=\alpha_{2} g_{2}=\alpha_{3} g_{3}$. Consequently

$$
\begin{aligned}
T C N_{2}= & \left\{\alpha_{2}\left(c_{13}+c_{23}+2\right)+2\left(1-\alpha_{2}\right)\right\} g_{2} \\
= & \left\{\frac{\alpha_{3} g_{3}}{g_{2}}\left(c_{13}+c_{23}+2\right)+2 \frac{g_{2}-\alpha_{3} g_{3}}{g_{2}}\right\} g_{2} \\
= & \alpha_{3} g_{3}\left(c_{13}+c_{23}+2\right)+2\left(g_{2}-\alpha_{3} g_{3}\right) \\
= & \alpha_{3} g_{3}\left(c_{13}+c_{23}\right)+2 g_{2} \\
= & \alpha_{3} g_{3}\left(c_{13}+c_{23}\right)+2\left(g_{3}-\alpha_{3} g_{3}\right) \\
= & \left\{\alpha_{3}\left(c_{13}+c_{23}\right)+2\left(1-\alpha_{3}\right)\right\} g_{3}=T C N_{3} \\
B A F_{2}^{u p p e r} & =\frac{\alpha_{2}\left(c_{23}+1\right)+\left(1-\alpha_{2}\right)}{\alpha_{2}\left(c_{13}+c_{23}+2\right)+2\left(1-\alpha_{2}\right)} \\
& =\frac{\frac{\alpha_{3}}{\alpha_{2}}\left\{\alpha_{2}\left(c_{23}+1\right)+\left(1-\alpha_{2}\right)\right\}}{\frac{\alpha_{3}}{\alpha_{2}}\left\{\alpha_{2}\left(c_{13}+c_{23}+2\right)+2\left(1-\alpha_{2}\right)\right\}} \\
& =\frac{\alpha_{3} c_{23}+\alpha_{3}+\left(\frac{\alpha_{3}}{\alpha_{2}}-\alpha_{3}\right)}{\alpha_{3}\left(c_{13}+c_{23}\right)+2 \alpha_{3}+2\left(\frac{\alpha_{3}}{\alpha_{2}}-\alpha_{3}\right)} \\
& =\frac{\alpha_{3} c_{23}+\left(1-\alpha_{3}\right)}{\alpha_{3}\left(c_{13}+c_{23}\right)+2\left(1-\alpha_{3}\right)}=B A F_{3}^{u p p e r} \\
& =\frac{\alpha_{3} c_{23}+\frac{\alpha_{3}}{\alpha_{2}}}{\alpha_{3}\left(c_{13}+c_{23}\right)+2 \frac{\alpha_{3}}{\alpha_{2}}}=\frac{\alpha_{3}}{\alpha_{3}\left(c_{13}+c_{23}\right)}+2 \frac{g_{2}}{g_{3}}
\end{aligned}
$$

\section{Resolving array CN scaling by approximate ploidy calculation}

Given the position of $(1,1)$ integer copies in a grid plot of noise-free array CNs, subclonal cell fractions and integer $\mathrm{CNs}$ can be derived for some segments and subclones in fortunate samples under certain assumptions. Unfortunately, the scaling (the position of $(1,1)$ ) of array $\mathrm{CNs}$ is generally unknown. By calculating subclone specific ploidies for each potential position of $(1,1)$, FACS plots can sometimes help us resolve the scaling issue (Figure 5).

This is how we estimate the ploidy of a selected subclone A under properties 1 and 2 and assumptions 1 and 2. Given the potential scale factor $g_{i}=1$, subclonal integer CNs of A can be estimated for each segment $j$ on lattice points of subclone A's grid plot by $\hat{c}_{1 j}=\left(a_{1 j}-g_{i-1}\right) /$ $\left(g_{i}-g_{i-1}\right), \hat{c}_{2 j}=\left(a_{2 j}-g_{i-1}\right) /\left(g_{i}-g_{i-1}\right)$, where $\vec{a}_{j}=\left(a_{1 j}, a_{2 j}\right)$ are minor and major array CNs.

If

Property 4: The fraction of the genome which cannot be resolved for integer $\mathrm{CNs}$ in subclone $\mathrm{A}$ is negligible with respect to the subclone's average $\mathrm{CN}$. then we can estimate the ploidy of subclone A by summing up these estimated integer CNs to $\sum_{j}\left[l_{j}\left(\hat{c}_{1 j}+\hat{c}_{2 j}\right)\right]$, where $l_{j}$ is the genomic length of segment $j$, and dividing the result by $\sum_{j} l_{j}$.

If at least one of the following holds, approximate overall ploidy estimates (across all the tumor cells) can be calculated from the array CNs for each potential position of $(1,1)$, and an independent overall ploidy estimate from, for example, FACS runs may help resolve the array $\mathrm{CN}$ scaling.

Property 5: The fraction of the genome which cannot be resolved for integer CNs (via subclones) is negligible with respect to the sample's average $\mathrm{CN}$.

Assumption 5: The average CN across the part of the genome which can be assessed for integer CNs (via subclones) is similar to the average $\mathrm{CN}$ across the rest of the genome.

This is how, under either property 5 or assumption 5 , we estimate overall ploidy in a sample with one evident subclone A. Given the potential scale factor $g_{i}=1$, the subclone A cell fraction is $\alpha=\left(g_{i}-g_{i-1}\right) / g_{i}$. Let:

$$
\text { overall ploidy }=\left(\frac{\alpha}{p}\right) \sum_{j}\left[\pi_{i}\left(\hat{c}_{1 j}+\hat{c}_{2 j}\right)\right]+2\left(\frac{1-\alpha}{p}\right)
$$

where $\pi_{j}$ is the fraction of the genome associated with segment $j$ and $p$ is a pathologist's estimate of sample purity. The relative size of subclone A among the tumor cells, $\alpha / p$, is also known as the subclone's ccf.

In samples with one evident subclone $\mathrm{A}$ as well as evidence of a larger subclone, $\mathrm{C}$, we may refine the overall ploidy estimate with the integer $\mathrm{CN}$ estimates mentioned earlier.

VAFs can sometimes help deduce the scaling of array CNs In this section we use the example in Figure 4 to explain the use of mutation VAFs to deduce the scaling of array CNs. This procedure again requires a set of subjectively chosen assumptions and only works under fortunate circumstances.

A grid plot of simulated, noise- and bias-free array $\mathrm{CNs}$ is shown in Figure 4a. The scale of the array CNs is unknown. The four colored points suggest the grid pattern drawn for a subclone $\mathrm{A}$, but it is unknown whether $(1,1)$ integer copies happen at $\left(g_{2}, g_{2}\right),\left(g_{3}, g_{3}\right)$ or $\left(g_{4}, g_{4}\right)$. The three scenarios areis illustrated in Figure b-d, which all have one colored column for each of the colored genome segments in Figure 4a. Equally between the panels, two of the segments have mutations on them (red crosses), with VAFs as shown on the y-axes (simulated without noise or bias). 
Each different scaling suggests different integer CNs $\left(c_{1}, c_{2}\right)$ for the segments (labels on $\mathrm{x}$-axes). For example, if $g_{2}=1$ (Figure $4 \mathrm{~b}$ ), the blue segment must have integer CNs $(2,2)$ in subclone A. Given a pair of integer CNs $\left(c_{1}\right.$, $c_{2}$ ), expected VAF levels can be derived under certain assumptions.

If

Property 6: A mutation sits on a segment that falls on a lattice point of a subclone A.

and we assume

Assumption 6: Mutations sit on a number of the $c_{1}+c_{2}$ local chromosomal copies in subclone A cells only.

in addition to relying on properties and assumptions 1 to 2, then we would expect VAF levels only in $\{\alpha c / D, c=1$, $\left.2, \ldots, c_{1}+c_{2}\right\}$, where $\alpha$ is the cell fraction of subclone A, and $D$ is the total (minor + major) average $\mathrm{CN}$ at the mutation's genomic position, $D=\alpha\left(c_{1}+c_{2}\right)+2(1-\alpha)$. Under these circumstances, and if $g_{2}=1$ (Figure $4 \mathrm{~b}$ ), the mutation on the blue segment in the example would sit on 1,2 , 3 or 4 of the $2+2$ chromosomal copies. The four corresponding expected VAF levels, simulated with sample purity $90 \%$, have been drawn as continuous, horizontal lines. The other scenarios, $g_{3}=1$ (Figure 4c) and $g_{4}=1$ (Figure $4 \mathrm{~d}$ ), suggest other VAF levels (continuous, horizontal lines).

If the sample purity $p$ is known (for example, from a pathologist's examination) and if, instead of assumption 6, we assume

Assumption 7: Mutations sit on one or both of the chromosomal copies of all tumor cells other than subclone A.

then we would expect VAF levels only in $\{(p-\alpha) c / D$, $c=1,2\}$. For mutations present in both subclone A cells and all other tumor cells, assume

Assumption 8: Mutations sit on a number of the $c_{1}+c_{2}$ local chromosomal copies in subclone A cells, and on one or both of the chromosomal copies of all tumor cells other than subclone A.

We call such mutations clonal, and for these we expect VAF levels only in $\left\{(\alpha c+(p-\alpha) d) / D, c=1,2, \ldots, c_{1}+c_{2}\right.$, $d=1,2\}$. If $g_{2}=1$ (Figure $4 \mathrm{~b}$ ), assumptions 7 and 8 and a purity of $90 \%$ give the 10 expected VAF levels drawn as dashed horizontal lines for the blue segment.

Pretending that assumptions 6 to 8 cover all possible locations of mutations on segments that fall on the grid plot lattice points fulfilling conditions 1 and 2, scenario $g_{4}=1$ (Figure $4 \mathrm{~d}$ ) can be ruled out - one mutation VAF in inexplicable as it does not coincide with a horizontal line. If we make the assumption that

Assumption 9: Mutations are heterozygous and present on all the copies of its homologue (thick continuous or dashed lines).

we can also rule out the scenario $g_{2}=1$ (Figure $4 \mathrm{~b}$ ) and fix the average $\mathrm{CNs}$ of Figure $4 \mathrm{c}$ for this sample.
Some samples have mutations which can help resolve the array $\mathrm{CN}$ scaling, like this. Other samples may have too few mutations, even in this optimal world with no noise in VAFs or segmented $\mathrm{CN}$ data.

\section{SNP array preprocessing and segmentation}

Genome-wide SNP analysis of tumor and matched normal samples was performed at AROS Applied Biotechnologies a/s (Aarhus, Denmark) on Affymetrix Genome-Wide Human SNP Arrays 6.0 (Affymetrix, Santa Clara, CA, USA) following the manufacturer's instructions, with the 52 tumor samples and the 29 available matched normal samples. The arrays were preprocessed with the ASCRMAv2 single-array method in the aroma.affymetrix $\mathrm{R}$ package [30,31], and further adjusted for SNP-specific allelic crosstalk with CalMaTe [32]. Total (signal A plus signal B) tumor SNP array signals were normalized (divided by) towards total SNP array signals of matched normal samples where available, or otherwise position-specific median total SNP array signals across the normal samples, giving TCNs for all tumors. BAFs were obtained and processed using TumorBoost. Allele-specific $\mathrm{CN}$ segments were identified from TCNs and BAFs with the paired or nonpaired PSCBS method [33] for samples with or without a matched normal sample. After this step we have a minor and a major array $\mathrm{CN}$ for each segment, equal to the median $\mathrm{TCN}\left(1-\mathrm{BAF}_{\text {upper }}\right)$ and $\mathrm{TCN}\left(\mathrm{BAF}_{\text {upper }}\right)$ across the SNPs in the segment. Two arrays failed this preprocessing.

The segmented array CNs were refined with HAPSEG [34], which phases the SNP alleles by comparing the sample-specific SNP data to large databases of normal sample SNP datasets. We let HAPSEG join up the adjacent segments we supplied with similar $\mathrm{CNs}$ to a limited extent (seg.merge.thresh $=1$ or $10^{-10}$ for different samples). HAPSEG significantly reduced $\mathrm{CN}$ bias in segments with allelic balance, which originally occurred because segment BAFs were estimated by the median distance between individual SNP BAF levels and 0.5, which is $>0$ even for segments with allelic balance. It also rescales the segment CNs so that they average to 1 for single homologues. The resulting homologue-specific segment $\mathrm{CNs}$ are referred to as array $\mathrm{CNs}$ throughout this paper.

All data analyses in this study were made with $R$ [35] unless otherwise stated.

\section{WES variant detection}

DNA was extracted using the DNeasy Blood and Tissue $\mathrm{Kit}^{\circ}$ (Qiagen, Venlo, Netherlands) following the manufacturer's instructions. DNA concentration was measured using the NanoDrop 1000 instrument (Thermo Scientific, Waltham, MA, USA). Whole exome sequencing was performed at DNAVision (Gosselies, Belgium). Genomic libraries from the tumor and matched normal samples were generated using the SureSelectXT Reagent 
Kit HSQ (Agilent Technologies, Santa Clara, CA, USA) following the manufacturer's instructions. Enrichment was performed using the SureSelectXT Human All Exon V4 + UTRs kit (Agilent) following the manufacturer's instructions.

Exome read alignment, filtering, variant calling and annotation were performed as follows. Cutadapt 1.1 [36] was used for quality-based adaptor trimming, sequence reads were aligned to the GRCh37/hg19 human reference genome using bwa-aln 0.7.7-r441 [37] and duplicate reads marked using Picard tools [38]. Aligned reads for each tumor-normal sample pair were combined into one alignment file in BAM format, followed by local indel realignment and base quality recalibration using the Genome Analysis Tool Kit (GATK) software [39]. The MuTect 2.7-1-g42d771f [40] program was used to identify somatic point mutations. Predictions not labeled as CERE$\mathrm{JECT}^{1}$ were accepted as confident somatic mutation predictions and considered for subsequent downstream validation and analysis steps. Variant annotation was performed using the Oncotator web-based service [41]. VAFs denote the number of reads with the detected variant as a fraction of all reads at the corresponding genomic position.

\section{Scaling bias in SNP array B allele fractions}

Grid plot skewness (Figure 7) is adjusted for as follows.

We assume that an unknown fraction $\alpha$ of the (germline-contaminated) tumor sample contributes the most visible, regular $\mathrm{CN}$ grid in the plot, and refer to this as our main subclone A. Note that subclone A may be all the tumor cells in the sample, in which case $\alpha$ is the sample purity, or it may be a true subclone of tumor cells. Within subclone A, each true single homologue average $\mathrm{CN} e$ should, theoretically, follow:

$$
e \in\{(1-\alpha)+\alpha c, c=0,1,2, \ldots\},
$$

where $c$ refers to the integer $\mathrm{CNs}$ in subclone $\mathrm{A}$. We model the observed minor and major array $\mathrm{CNs}$ $\vec{a}=\left(a_{1}, a_{2}\right)$ of an arbitrary CN segment as if they have been subject to a plane rotation $F=\left(\begin{array}{ll}f_{11} & f_{12} \\ f_{21} & f_{22}\end{array}\right)$ of the average CNs $\vec{e}=\left(e_{1}, e_{2}\right)$, which are functions of the integer CNs $\vec{c}=\left(c_{1}, c_{2}\right)$ in A: $\vec{a}=F \vec{e}$, that is:

$$
\left(\begin{array}{l}
a_{1} \\
a_{2}
\end{array}\right)=\left(\begin{array}{ll}
f_{11} & f_{12} \\
f_{21} & f_{22}
\end{array}\right)\left(\begin{array}{l}
1-\alpha+\alpha c_{1} \\
1-\alpha+\alpha c_{2}
\end{array}\right) .
$$

Our aim is to estimate the rotation matrix $F$, assumed to be common to all the $\mathrm{CN}$ segments in the sample, and hence to derive the skewness adjusted array CNs $\vec{a}^{\prime}=\hat{F}-1 \vec{a}$. The matrix $F$ involves a scale factor dependent on the unknown fraction $\alpha$. For the sake of grid rotation we use the maximum $\alpha$ that fits the array CNs. This is the scaling scenario which corresponds to the smallest possible position of $(1,1)$. (The final scaling step for estimation of $\alpha$ and the integer CNs $\left\{\left(c_{1}, c_{2}\right)\right\}$ is based on observed VAFs as described below.)

We find M-estimates of $\alpha$ and the matrix $F$ numerically $[42,43]$ by minimizing of the sum of the distances from each $\mathrm{CN}$ segment $\vec{a}$ to its closest skewed lattice point $F \vec{e}$ weighted by the robust Tukey function and the length of the genome segment corresponding to each $\vec{a}$. An M-estimator [44,45] is a generalization of the maximum likelihood (ML-) estimator. It minimizes the summed values of a function $\rho, \hat{F}=\operatorname{argmin}_{F} \sum_{i=1}^{n} \rho\left(r_{i}\right)$, where $\rho$ is similar to but not necessarily a likelihood function. We let $r_{i}=w_{i}\left|\vec{a}_{i}-F \vec{e}_{i}\right|$ for each segment $i$, where $F \vec{e}_{i}$ is the closest lattice point to $\vec{a}_{i}$, and $w_{i}$ is a weight $\leq 1$ determined by the length $l_{i}$ of segment $i$ (typically $w_{i}=1-e^{-l_{i} / 500000}$ ) to downweight short segments (typically $<1 \mathrm{Mb}$ ), which might have less reliable array CNs. Tukey's $\rho$ function truncates its input in a smooth fashion, so that observations far away have a limited influence on our estimate. In this way, we avoid segments that violate the grid (for example, because they belong to a different subclone), blurring our estimate of $F$. Figure 7c shows a resulting grid plot after grid rotation.

Starting values $\alpha_{0}$ and $F_{0}$ for the grid rotation are derived in two steps. Step 1 estimates a pre-start matrix $F^{\prime}=\left(\begin{array}{ll}f_{11}^{\prime} & f_{12}^{\prime} \\ f_{21}^{\prime} & f_{22}^{\prime}\end{array}\right)$, with $F^{\prime}=\alpha F$ so that a pre-rotation $\left(F^{\prime}\right)^{-1} \vec{a}$ gives a non-skewed grid plot with unit increments between vertical and horizontal lattice points (Figure 7b):

$$
\begin{aligned}
\left(\begin{array}{l}
a_{1} \\
a_{2}
\end{array}\right) & =\frac{1}{\alpha} F^{\prime}\left(\begin{array}{c}
1-\alpha+\alpha c_{1} \\
1-\alpha+\alpha c_{2}
\end{array}\right) \\
& =F^{\prime}\left(\begin{array}{c}
\frac{1-\alpha}{\alpha}+c_{1} \\
\frac{1-\alpha}{\alpha}+c_{2}
\end{array}\right), \\
& \left(F^{\prime}\right)^{-1}\left(\begin{array}{c}
a_{1} \\
a_{2}
\end{array}\right)=\left(\begin{array}{c}
\frac{1-\alpha}{\alpha}+c_{1} \\
\frac{1-\alpha}{\alpha}+c_{2}
\end{array}\right) .
\end{aligned}
$$

Rough settings are collected by manually selecting informative clusters in the skewed grid plot (Figure 7a):

$d_{y}=$ vertical component of the distance between two 'vertically' consecutive clusters $d_{x}=$ horizontal component of the distance between two 'horizontally' consecutive clusters slope $_{y}=$ slope of the line through two 'vertically' consecutive clusters 
slope $_{x}=$ slope of the line through two 'horizontally' consecutive clusters

Let $\left(c_{1 j}, c_{2 j}\right)$ and $\left(c_{1 j+1}, c_{2 j+1}\right)$ be the (unknown) integer CNs of subclone A seen as two consecutive, 'vertical' clusters in the skewed grid plot, such that $c_{1 j}+1=c_{1 j+1}$ and $c_{2 j}=c_{2 j+1}$. Then

$$
\begin{aligned}
d_{y}= & \left(f_{11}^{\prime}\left[\frac{1-\alpha}{\alpha}+c_{1 j+1}\right]+f_{12}^{\prime}\left[\frac{1-\alpha}{\alpha}+c_{2 j+1}\right]\right) \\
& -\left(f_{11}^{\prime}\left[\frac{1-\alpha}{\alpha}+c_{1 j}\right]+f_{12}^{\prime}\left[\frac{1-\alpha}{\alpha}+c_{2 j}\right]\right)=f_{11}^{\prime}
\end{aligned}
$$

and similarly $d_{x}=f_{22}^{\prime}$, slope $y=f_{11}^{\prime} / f_{21}^{\prime}$ and slope $_{x}=f_{12}^{\prime} / f_{22}^{\prime}$. We estimate the pre-start matrix $F^{\prime}$ by:

$$
\left\{\begin{array}{c}
f_{11}^{\prime}=d_{y} \\
f_{22}^{\prime}=d_{x} \\
f_{21}^{\prime}=f_{11}^{\prime} / \text { slope }_{y} \\
f_{12}^{\prime}=f_{22}^{\prime} \text { slope }_{x} .
\end{array}\right.
$$

In step 2 we estimate $\alpha_{0}$, the maximum possible fraction $\alpha$ such that $0<\alpha \leq 1$. Starting from a selected lattice point with allelic balance a grid with step size one is imposed on the pre-rotated Figure $7 \mathrm{~b}$, stretching as close to zero as possible with all gridlines positive. Let the array $\mathrm{CN}$ levels of the first two horizontal gridlines be $\alpha_{i}$ and $\alpha_{i i}$. Using Equation 3 we derive $\alpha_{\max }^{x}=\left(a_{i i}-a_{i}\right) / a_{i i}$, and similarly for $\alpha_{\text {max }}^{y}$ from vertical gridlines. We set start values for the numerical optimization to $\alpha_{0}=\left(\alpha_{\max }^{x}+\alpha_{\max }^{y}\right) / 2$ and $F_{0}=F^{\prime} / \alpha_{0}$.

What is the origin of the grid bias, the skewness? We further investigate the SNP array components from which array $\mathrm{CNs}$ are computed: TCNs and BAFs. Note that the BAFs here refer to the upper BAF of each segment, which is always between 0.5 and 1 .

We plot the observed total array CNs $a_{1}+a_{2}$ towards the rotated, supposedly unbiased $a_{1}^{\prime}+a_{2}^{\prime}$ (Figure 13a) as well as observed (upper) BAFs $a_{2} /\left(a_{1}+a_{2}\right)$ towards the rotated (upper) BAFs $a_{2}^{\prime} /\left(a_{1}^{\prime}+a_{2}^{\prime}\right)$ (Figure 13b). Assuming the rotated $\mathrm{CNs}$ are truly proportional to the true average $\mathrm{CNs}$, the graph suggests the original total $\mathrm{CNs}$ (since proportional to rotated $\mathrm{CNs}$ ) are indeed also proportional to the true average CNs. The biased total array $\mathrm{CNs}$ and the rotated ones have different scale factors, but that cannot be the cause or adjustment for skewness. In Figure 13a single homologue (minor and major) original
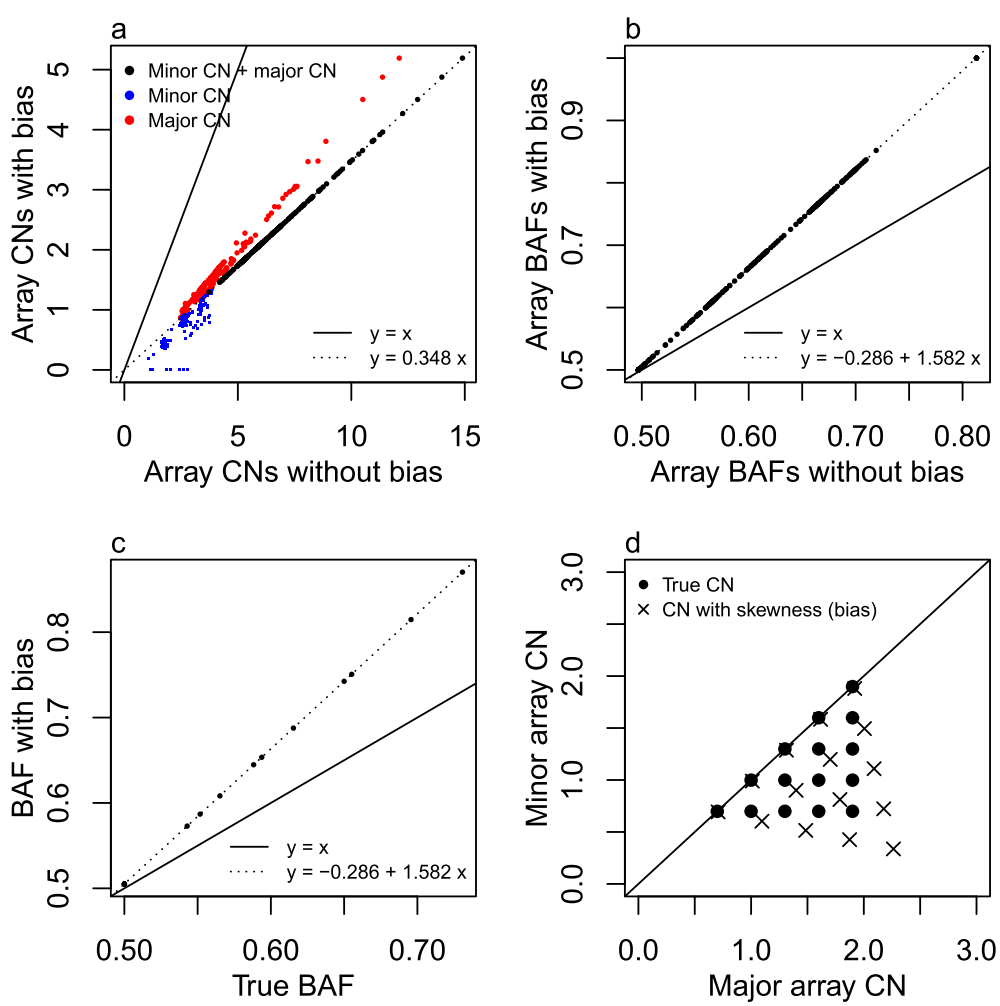

Figure 13 Origin of skewness. (a) Sample 45 before versus after rotation TCNs are exactly proportional, whereas minor or major CNs show a more complicated difference. (b) Sample 45 BAFs before rotation (upper) carry a 0.5 centered scaling bias compared to after rotation (upper), a bias which causes grid plot skewness. (c) Imposed BAF bias on simulated array data in (d). (d) Grid plots of true and BAF-induced biased array CNs in a simulated dataset. 
and rotated $\mathrm{CNs}$ are not just proportional to each other, but seem subject to bias related to single $\mathrm{CN}$ magnitude. Indeed, Figure 13b suggests BAFs carry scaling bias centered at 0.5. If had plotted the lower BAFs instead (which are between 0 and 0.5 ), Figure $13 \mathrm{~b}$ would have shown points on the bottom left extension of the dotted line. Either way the deviation between BAFs before and after rotation is small for BAFs close to 0.5 and larger further away. To investigate whether such a BAF bias may cause grid plot skewness, we simulate a set of array CNs as in Figure 1, and derive its true BAFs. We then created a biased dataset with total array CNs as in Figure 1 but with BAFs biased (Figure 13c) according to the estimated linear model in the real dataset (Figure 13b). Plotting both the true and the biased simulated array CNs in Figure $13 \mathrm{~d}$ reveals that a 0.5 centered scaling bias of BAFs may indeed cause skewness in grid plots.

The connection between grid plot skewness and bias in BAFs introduces a relationship between the expected association in Figure 13, $B A F_{\text {observed }}=k+l \times B A F_{\text {true }}$, and the rotation matrix $F$ up to a scaling constant $C$ :

$$
F=C\left(\begin{array}{cc}
1-k & 1-k-l \\
k & k+l
\end{array}\right)
$$

For the example sample 45 we estimated $B A F_{\text {observed }}=$ $-0.286+1.582 B A F_{\text {rotated }}$, so we should have $F$ proportional to $\left(\begin{array}{cc}1.286 & -0.286 \\ -0.296 & 1.296\end{array}\right)$, and from the grid rotation we indeed estimated:

$$
F=\left(\begin{array}{cc}
0.447 & -0.103 \\
-0.101 & 0.452
\end{array}\right)=0.349\left(\begin{array}{cc}
1.280 & -0.294 \\
-0.289 & 1.295
\end{array}\right)
$$

Equation 4 imposes the restriction $f_{11}+f_{21}=f_{12}+f_{22}$ on $F$, which can sometimes help the numerical optimization.

The BAF bias causes segments with very low minor CNs to get upper BAFs biased down to 1 in the array preprocessing steps. Such segments will appear as a horizontal bottom line in original grid plots, and a sloped bottom line after grid rotation. We believe that these segments should have had a constant minor array $\mathrm{CN}$, and so we project the corresponding grid plot points vertically down to the observed bottom line of constant minor array CNs when the latter is evident.

Unless otherwise stated, we refer to array CNs as rotated array $\mathrm{CNs}$ after projection of bottom sloped line array CNs, and we drop the prime from $\vec{a}^{\prime}$.

\section{Estimation of subclonal architecture, cell fractions and integer CNs in RESPONSIFY samples}

The cell fraction and integer $\mathrm{CNs}$ are estimated for type A segments of cells in the most evident subclone A. According to our assumptions all other cells are diploid in these segments.
$\mathrm{CN}$ alteration in type $\mathrm{B}$ segments may be due to further $\mathrm{CN}$ alteration in daughter subclones of $\mathrm{A}$ or by $\mathrm{CN}$ alteration in subclones independent of A. For some segments which have many mutations we can deduce the subclonal origin via the mutation VAFs as in the following example. In Figure 11, VAFs in type A segments (blue) match the expected VAF levels of subclone A $\left(\left\{\alpha c / D, c=c_{1}, c_{2}\right\}\right.$ with notation as above but now referring to estimates from real data), which is reassuring for our analyses. VAFs in type B segments (pink) also match expected VAF levels of subclone A $\left(\left\{\alpha c / D, c=c_{1}, c_{2}\right\}\right.$ with $c_{1}, c_{2}$ being fractional rather than integer $\mathrm{CNs}$ ). This suggests the true CNs indeed meet the fractional $c_{1}, c_{2}$ in a fraction $\alpha$ of the sample cells, which in turn suggests subclone A has daughter subclones with further $\mathrm{CN}$ alteration in these segments.

For 25 of the 48 samples resolved for array scaling we identified an additional subclone $\mathrm{C}$ by the existence of a lower grid pattern in the grid plot (see Materials and methods). In these cases we call the lower grid pattern segments type $C$ segments, estimate the subclone $C$ cell fraction approximately, assign integer $\mathrm{CNs}(0,1)$ to the subclone $\mathrm{C}$ type $\mathrm{C}$ segments, and estimate integer $\mathrm{CNs}$ of subclone $\mathrm{A}$ in the type $\mathrm{C}$ segments via Equation 2.

All our samples lack regular grid patterns in high array $\mathrm{CN}$ segments (red in Figure 8). We call these type D segments. Their $\mathrm{CN}$ alteration may take place in subclone A (but the grid is not regular because the proportionality between array $\mathrm{CNs}$ and average $\mathrm{CNs}$ breaks down with high SNP array intensities), in subclone $\mathrm{C}$ or in any other subclone. As for type B segments, some segments which have many mutations can be assigned to a subclone via the mutation VAFs. In the example of Figure 11, the VAFs in type D segments (red) match the expected VAF level of the minor homologue of subclone $\mathrm{A}\left(\left\{\alpha c_{1} / D\right\}\right.$ where $c_{1}$ is a fractional $\mathrm{CN}$. This suggests that any $\mathrm{CN}$ alteration in the minor homologue takes place in subclone A.

\section{Clonal or subclonal mutations in RESPONSIFY samples}

Continued from the 'Clonal or subclonal mutations' section in Results. Segment types are exemplified in Figure 8.

For samples with only one identified subclone A, we classify each type A mutation as clonal if the CI falls above $\left(\alpha c_{1}+(p-\alpha)\right) / D-\delta$, with $\delta=0.1$ (non-inferiority test at significance level 5\%), $\alpha, c_{1}$ and $c_{2}$ estimates for subclone $\mathrm{A}$ and $D$ the estimate of the local minor + major average $\mathrm{CN}$. We classify a mutation as subclonal if its CI falls below $\left(\alpha c_{1}+(p-\alpha)\right) / D$ (one-sided inferiority test at significance level $5 \%$ ). Some mutations will be called ambiguous. For subclonal mutations we further test whether they sit on subclone A or not, with equality tests significant if the CI falls within $\alpha c_{1} / D \pm \delta$ or $\alpha c_{2} / D \pm \delta$. 
If, in addition to a most evident subclone $\mathrm{A}$, a sample has another identified subclone $\mathrm{C}$ in a fraction $\gamma$ of the cells, we assess whether the subclonal type A mutations seem to sit on A only $\left(\mathrm{CI} \in \alpha c_{1} / D \pm \delta\right.$ or $\mathrm{CI} \in$ $\left.\alpha c_{2} / D \pm \delta\right)$, on $\mathrm{C}$ only $(\mathrm{CI} \in \gamma / D \pm \delta)$ or on both $\mathrm{A}$ and $\mathrm{C}\left(\mathrm{CI} \in\left(\alpha c_{1}+\gamma\right) / D \pm \delta\right.$ or $\left.\mathrm{CI} \in\left(\alpha c_{2}+\gamma\right) / D \pm \delta\right)$. The extended procedure creates more ambiguous mutations, since we only allow non-ambiguous classifications.

Mutations of type B or D are classified like A mutations (so that equivalence to a specific subclonal expected VAF level suggests further daughter subclones with $\mathrm{CN}$ alteration in $\mathrm{B}$ segments) unless the sample has an identified subclone $C$. In case of the latter, segments of type B or D may have (I) integer CNs $\left(c_{1 a}, c_{2 a}\right)$ in subclone $\mathrm{A}$ and $(1,1)$ in other tumor cells, or, they may have (II) integer CNs $\left(c_{1 c}, c_{2 c}\right)$ in subclone $\mathrm{C}$ and $(1,1)$ in other tumor cells, or something else. We classify the type $\mathrm{B}$ and $\mathrm{D}$ mutations as outlined for type A mutations (based on I) in parallel with an analogous procedure based on II. Only mutations for which both classifications agree are finally assigned a class different from ambiguous.

Type C mutations are classified as type B or D ones, except in samples with an identified subclone $C$, for which we set the minor homologue integer $\mathrm{CNs}$ in subclone $C$ to $(0,1)$ instead of $(1,1)$, and adjust the subclone A integer $\mathrm{CNs}$ according to Equation 2.

Approximately $35 \%$ of the 52 RESPONSIFY samples have the majority of type $B$ and D VAFs matching expected VAF levels for $\mathrm{CN}$ alteration in subclone A. Most samples have no or only a handful of mutations in type C segments.

\section{Grid plots reveal bias}

This section illustrates the benefit of two-dimensional grid plots compared with one-dimensional histograms, in order to reveal bias in array CNs.

Each point in the original (Figure 14a) and rotated (Figure 14b) array $\mathrm{CN}$ grid plots shows the minor and major array $\mathrm{CN}$ of a genome segment. In the Absolute software, the minor and major array $\mathrm{CNs}$ are pooled and shown in (one-dimensional) histograms (Figure 14c for original and Figure 14d for rotated array $\mathrm{CNs}$ ) with heights proportional to segment lengths. Hence, each segment is represented twice in the histograms - once with the minor array $\mathrm{CN}$ and once with the major. Segments with subclone A two-dimensional $\mathrm{CN}$ estimates $(1,1),(2,2),(0,2)$ and $(1,2)$ have been colored equally in all four panels (black, red, blue, green). The cyan segments have non-integer CNs (between 1 and 2) with respect to
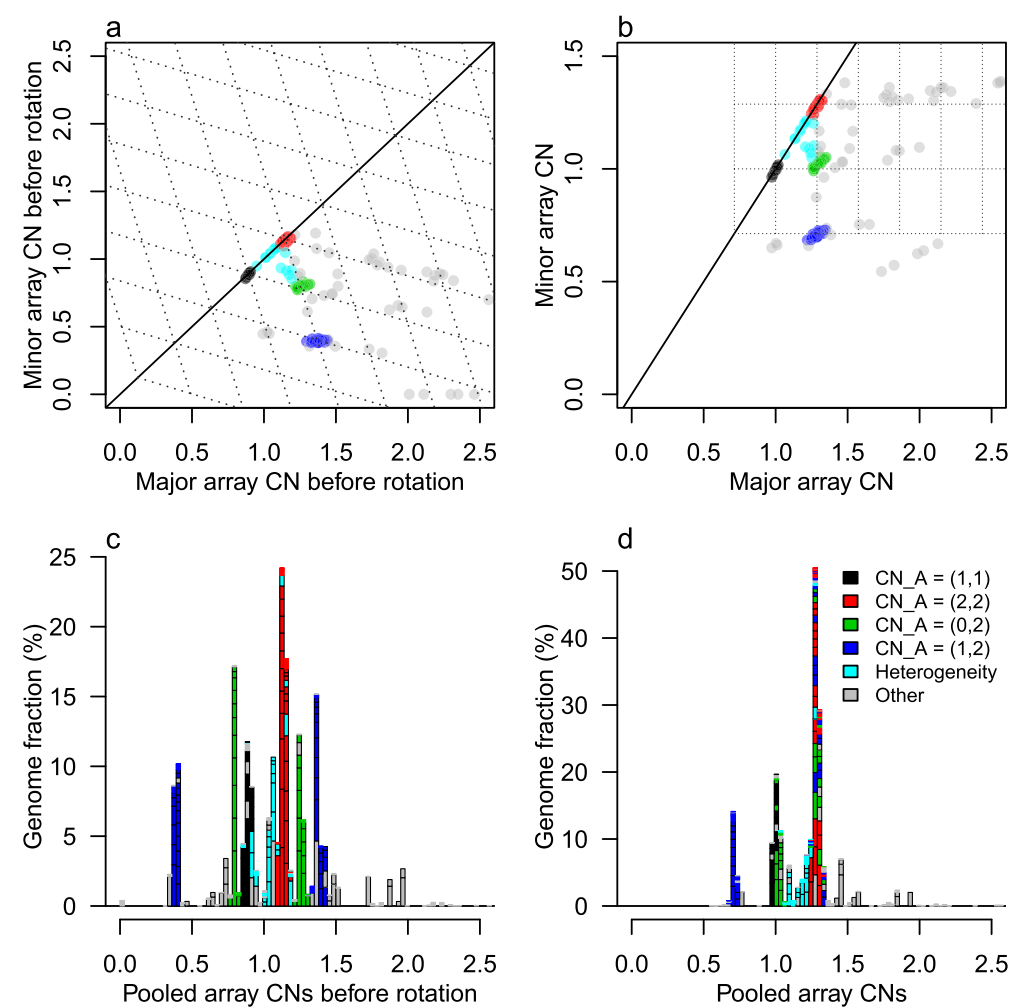

Figure 14 Sample 11 grid plots and Absolute histograms. (a) Original grid plot. (b) Grid plot after rotation. (c) Absolute histogram based on original array CNs. (d) Absolute histogram based on rotated array CNs. Note that all plots show the array CNs scaled to equal average CNs, so that the level one (1) corresponds to normal, single haplotype CNs. 
subclone A. They may have further heterogeneity within subclone A or originating from a subclone independent of A. Absolute searches for equally interspaced peak centers in the histogram with a maximum likelihood algorithm, and each peak is assigned an integer $\mathrm{CN}$ estimate. Segments that fall significantly far from their closest peak centers are classified as subclonal, under the assumptions that (i) only one pattern of equally interspaced peaks can occur, and (ii) the pattern reflects the clonal $\mathrm{CNs}$ of all tumor cells in the sample. According to the $\mathrm{CN}$ coloring in Figure 14, three colored histogram peaks are expected: $\mathrm{CN}=0$ (blue), $\mathrm{CN}=1$ (black, green) and $\mathrm{CN}=2$ (red, blue, green). In the histogram of original array CNs (Figure 14a) it is hardly possibly to identify the three $\mathrm{CN}$ levels, their centers are not equally interspaced, and non-integer $\mathrm{CNs}$ (cyan) are intermixed with the integer CNs. Since all our samples have skewness, Absolute did not assign integer CNs optimally.

\section{Data and implementation}

The preprocessed array $\mathrm{CN}$ data for the six samples discussed in this paper are available as Additional files 1,2, $3,4,5$ and 6, while the Oncotator annotated Mutect variants for two of these samples are available in Additional files 7 and 8. A CRAN package to identify grid patterns, perform our grid rotation algorithm and calculate the ITH endpoint will be available shortly with full documentation under the name 'Gridith'. A "Gridith" beta version is available at https://github.com/fcaramia/GRIDITH.

\section{Additional files}

\section{Additional file 1: Sample 5 preprocessed segment array CNs. Additional file 2: Sample 9 preprocessed segment array CNs. Additional file 3: Sample 11 preprocessed segment array CNs. Additional file 4: Sample 16 preprocessed segment array CNs. Additional file 5: Sample 29 preprocessed segment array CNs. Additional file 6: Sample 45 preprocessed segment array CNs. Additional file 7: Sample 5 mutect variants oncotator annotated mindepth 10 .}

Additional file 8: Sample 16 mutect variants oncotator annotated mindepth 10 .

\section{Abbreviations}

BAF: B allele fraction; ccf: cancer cell fraction; Cl: confidence interval; CN: copy number; FACS: fluorescence-activated cell sorting; ITH: intra-tumor heterogeneity; LOH: loss of heterozygosity; SNP: single nucleotide polymorphism; TCN: total copy number; VAF: variant allele fraction; WES: whole exome sequencing; WGS: whole genome sequencing.

\section{Competing interests}

IML conducted this work while on leave from the commercial consultancy company Statisticon AB, Sweden.

\section{Authors' contributions}

ShL, CSW, TPS and IML conceived and designed the study. DF, RS, SG, SiL, PN and CSo provided study materials. AR and NK ran the FACS analyses. RS read the tumors for purity. FC and $J \mathrm{~L}$ carried out the exome read alignment, filtering, variant calling and annotation. IML carried out preprocessing and segmentation of SNP array data. IML and TPS performed the statistical analysis. MS and SH provided intellectual input to the data analysis. PS provided help to interpret the data analysis results with respect to HER2-enriched tumors. IML and TPS prepared the initial draft manuscript. All authors read and approved the final manuscript.

\section{Acknowledgements}

We thank Henrik Bengtsson at University of California, San Fransisco, USA and Pierre Neuvial, CNRS, France for useful discussions on BAF bias, and Henrik also for advice on using the aroma.affymetrix and PSCBS R packages. We also like to acknowledge Marcus Mayrhofer at Uppsala University, Sweden for introducing us to two-dimensional patterns in SNP array data in the form of TAPS plots. This project was supported by European Union FP7 project 'RESPONSIFY' No 278659. ShL was supported by Cancer Council Victoria. TPS was supported by NHMRC Australia Fellowship.

\section{Author details}

${ }^{1}$ Bioinformatics Division, The Walter and Eliza Hall Institute of Medical Research, 1G Royal Parade, Parkville, VIC 3052, Australia. ${ }^{2}$ University of Melbourne, Melbourne, VIC 3010, Australia. ${ }^{3}$ Division of Research and Cancer Medicine, Peter MacCallum Cancer Centre, East Melbourne, VIC 3002, Australia. ${ }^{4}$ Breast Cancer Translational Research Laboratory, Institut Jules Bordet, Brussels, Belgium. ${ }^{5}$ Cancer Research UK, London Research Institute, Translational Cancer Therapeutics Laboratory, 44 Lincoln's Inn Fields, London WC2A 3LY, UK. 'Bioinformatics and BioStatistics, Cancer Research UK, Lincoln's Inn Fields, Holborn, London WC2A 3LY, UK. ${ }^{7}$ Translational Cancer Therapeutics Laboratory, UCL Cancer Institute, Paul O'Gorman Building, University College London, 72 Huntley Street, London WC1E 6DD, UK. ${ }^{8}$ German Breast Group (GBG), Neu Isenburg, Germany. ${ }^{9}$ Multidisciplinary Breast Centre and Gynaecological Oncology, KU Leuven, University of Leuven, Department of Oncology, B-3000 Leuven, Belgium. ${ }^{10}$ UCL Cancer Institute, Paul O'Gorman Building, University College London, 72 Huntley Street, London WC1E 6DD, UK. "Department of Mathematics and Statistics, University of Melbourne, Melbourne, VIC 3010, Australia.

Received: 2 May 2014 Accepted: 15 September 2014

Published online: 01 October 2014

\section{References}

1. Carter SL, Cibulskis K, Helman E, McKenna A, Shen H, Zack T, Laird PW, Onofrio RC, Winckler W, Weir BA, Beroukhim R, Pellman D, Levine DA, Lander ES, Meyerson M, Getz G: Absolute quantification of somatic DNA alterations in human cancer. Nat Biotechnol 2012, 30:413-421.

2. Durinck S, Ho C, Wang NJ, Liao W, Jakkula LR, Collinsson EA, Pons J, Chan S-W, Lam ET, Chu C, Park K, Hong S, Hur JS, Huh N, Neuhaus IM, Yu SS, Grekin RC, Mauro TM, Cleaver JE, Kwok P-Y, LeBoit PE, Getz G, Cibulskis K, Aster JC, Huang H, Purdom E, Li J, Bolund L, Arron ST, Gray JW, et al: Temporal dissection of tumorigenesis in primary cancers. Cancer Discov 2011, 1:137-143.

3. Gerlinger M, Rowan A, Horswell S, Larkin J, Endesfelder D, Gronroos E, Martinez P, Matthews N, Stewart A, Tarpey A, Varela I, Phillimore B, Begum S, McDonald NQ, Butler A, Jones D, Raine K, Latimer C, Santos CR, Nohadani M, Eklund AC, Spencer-Dene B, Clark G, Pickering L, Stamp G, Gore M, Szallasi Z, Downward J, Futreal PA, Swanton C: Intratumor heterogeneity and branched evolution revealed by spatial sequencing. NEJM 2012, 366:10.

4. Ha G, Roth A, Lai D: Integrative analysis of genome-wide loss of heterozygosity and mono-allelic expression at nucleotide resolution reveals disrupted pathways in triple negative breast cancer. Genome Res 2012, 22:1995-2007.

5. Landau DA, Carter SL, Stojanov P, McKenna A, Stevenson K, Lawrence MS, Sougnez C, Stewart C, Sivachenko A, Wang L, Wan Y, Zhang W, Shukla SA, Vartanov A, Fernandes SM, Saksena G, Cibulskis K, Tesar B, Gabriel S, Hacohen N, Meyerson M, Lander ES, Neuberg D, Brown JR, Getz G, Wu CJ: Evolution and impact of subclonal mutations in chromic lymphocytic leukemia. Cell 2013, 152:714-726.

6. Navin N, Krasnitz A, Rodgers L, Cook K, Meth J, Kendall J, Riggs M, Eberling Y, Troge J, Grubor V, Levy D, Lundin P, Månér S, Zetterberg A, Hicks J, Wigler M: Inferring tumor progression from genomic heterogeneity. Genome Res 2010, 20:68-80.

7. Navin N, Kendall J, Troge J, Andrews P, Rodgers L, McIndoo J, Cook K, Stepansky A, Levy D, Esposito D, Muthuswamy L, Krasnitz A, McCombie WR, 
Hicks J, Wigler M: Tumor evolution inferred by single-cell sequencing. Nature 2011, 472:90-94.

8. Nik-Zainal S, Van Loo P, Wedge DC, Alexandrov LB, Greenman CD, Wai Lau K, Raine K, Jones D, Marshall J, Ramakrishna M, Shlien A, Cooke SL, Hinton J, Menzies A, Stebbings LA, Leroy C, Jia M, Rance R, Mudie LJ, Gamble SJ, Stephens PJ, McLaren S, Tarpey PS, Papaemmanuil E, Davies HR, Varela I, McBride DJ, Bignell GR, Leung K, Butler AP, et al: The life history of 21 breast cancers. Cell 2012, 149:994-1007.

9. Papaemmanuil E, Gerstung M, Malcovati L, Tauro S, Gundem G, Van Loo P, Yoon CJ, Ellis P, Wedge DC, Pellagatti A, Shlien A, Groves MJ, Forbes SA, Raine K, Hinton J, Mudie LJ, McLaren S, Hardy C, Latimer C, Della Porta MG, O'Meara S, Ambaglio I, Galli A, Butler AP, Walldin G, Teague JW, Quek L, Sternberg A, Gambacorti-Passerini C, Cross NC, et al: Clinical and biological implications of driver mutations in myelodysplastic syndromes. Blood 2013, 122:3616-3627.

10. Shah SP, Morin RD, Khattra J, Prentice L, Pugh T, Burleigh A, Delaney A, Gelmon K, Guliany R, Senz J, Steidl C, Holt RA, Jones S, Sun M, Leung G, Moore R, Severson T, Taylor GA, Teschendorff AE, Tse K, Turashvili G, Varhol R, Warren RL, Watson P, Zhao Y, Caldas C, Huntsman D, Hirst M, Marra MA Aparicio S: Mutational evolution in a lobular breast tumor profiled at single nucleotide resolution. Nature 2009, 461:809-813.

11. Walter MJ, Shen D, Ding L, Shao J, Koboldt DC, Chen K, Larson DE, McLellan MD, Dooling D, Abbott R, Fulton R, Magrini V, Schmidt H, Kalicki-Veizer J, O'Laughlin M, Fan X, Grillot M, Witowski S, Heath S, Frater JL, Eades W, Tomasson M, Westervelt P, DiPersio JF, Link DC, Mardis ER, Ley TJ, Wilson RK, Graubert TA: Clonal architecture of secondary acute myeloid leukemia. N Engl J Med 2012, 366:1090-1098.

12. Chen $M$, Gunel $M$, Zhao H: SomatiCA: identifying, characterizing and quantifying somatic copy number aberrations from cancer genome sequencing data. PLoS One 2013, 8:e78143.

13. Xu Y, Muller P, Yuan Y, Gulukota J, Ji J: MAD bayes for tumor heterogeneity - feature allocation with exponential family sampling. Available at arXiv 2014, 1402.5090

14. Roth A, Khattra J, Yap D, Wan A, Laks E, Biele J, Ha G, Aparicio S, Bouchard-Cote A Shah SP: PyClone: statistical inference of clonal population structure in cancer. Nat Methods 2014, 11:396-398.

15. Jiao W, Vembu S, Deshwar AG, Stein L, Morris Q: Inferring clonal evolution of tumors from single nucleotide somatic mutations. BMC Bioinformatics 2014, 15:35.

16. Fischer A, Vasquez-Garcia I, Illingworth CJR, Mustonen V: High-definition reconstruction of clonal composition in cancer. Cell Rep 2014, 7:1740-1752.

17. Hajirasouliha I, Mahmoody A, Raphael BJ: A combinatorial approach for analyzing intra-tumor heterogeneity from high-throughput sequencing data. Bioinformatics 2014, 30:i78-i86.

18. Keats JJ, Chesi M, Egan JB, Garbitt VM, Palmer SE, Braggio E, Van Wier $S$, Blackburn PR, Baker AS, Dispenzieri A, Kumar S, Rajkumar SV, Carpten JD, Barrett M, Fonseca R, Stewart AK, Bergsagel PL: Clonal competition with alternating dominance in multiple myeloma. Blood 2012, 120:1067-1076.

19. Lee AJ, Endesfelder D, Rowan AJ, Walther A, Birkbak NJ, Futreal PA, Downward J, Szallasi Z, Tomlinson IP, Howell M, Kschischo M, Swanton C: Chromosomal instability confers intrinsic multidrug resistance. Cancer Res 2011, 71:1858-1870

20. RESPONSIFY. [http://www.responsify-fp7.eu/]

21. Rasmussen M, Sundström M, Göransson Kultima H, Botling J, Micke $P$, Birgitsson H, Glimelius B, Isaksson A: Allele-specific copy number analysis of tumor samples with aneuploidy and tumor heterogeneity. Genome Biol 2011, 12:R108.

22. Van Loo P, Nordgard $\mathrm{SH}$, Lingjærde $\mathrm{OC}$, Russnes $H G$, Rye $\mathrm{IH}$, Sun $\mathrm{W}$, Weigman VJ, Marynen P, Zetterberg A, Naume B, Perou CM: Allele-specific copy number analysis of tumors. Proc Natl Acad Sci U S A 2010, 107:39.

23. Pounds S, Cheng C, Mullighan C, Raimondi SC, Shurtleff S, Downing JR: Reference alignment of SNP microarray signals for copy number analysis of tumors. Bioinformatics 2009, 25:315-321.

24. Assié $G$, LaFramboise T, Platzer P, Bertherat J, Stratakis CA, Eng C: SNP arrays in heterogeneous tissue: highly accurate collection of both germline and somatic genetic information from unpaired single tumor samples. Am J Hum Genet 2008, 82:903-915.

25. Greenman CD, Bignell G, Butler A, Edkins A, Hinton J, Beare D, Swamy S, Santarius T, Chen L, Widaa S, Futreal PA, Stratton MR: PICNIC: an algorithm to predict absolute allelic copy number variation with microarray cancer data. Biostatistics 2010, 11:164-175.
26. Nancarrow DJ, Handoko HY, Stark MS, Whiteman DC, Hayward NK: SiDCoN: A tool to aid scoring of DNA copy number changes in SNP chip data. PLOS One 2007, 2:e1093.

27. Popova T, Manié E, Stoppa-Lyonnet D, Rigaill G, Barillot E, Stern MH: Genome Alteration Print (GAP): a tool to visualize and mine complex cancer genomic profiles obtained by SNP arrays. Genome Biol 2009, 10:R128.

28. Lange KL, Little RJA, Taylor JMG: Robust statistical modeling using the $t$ distribution. JASA 1989, 84:408

29. Lawrence MS, Stojanov P, Polak P, Kryukov GV, Cibulskis K, Silvachenko A Carter SL, Stewart C, Mermel CH, Roberts SA, Kiezun A, Hammerman PS, McKenna A, Drier Y, Zou L, Ramos AH, Pugh TJ, Stransky N, Helman E, Kim J, Sougnez C, Ambrogio L, Nickerson E, Shefler E, Cortés ML, Auclair D, Saksena G, Voet D, Noble M, DiCara D, et al: Mutational heterogeneity in cancer and the search for new cancer-associated genes. Nature 2013. doi:10.1038/nature12213.

30. Bengtsson H, Simpson K, Bullard J, Hansen K: aroma.affymetrix: A generic framework in $\mathrm{R}$ for analyzing small to very large Affymetrix data sets in bounded memory. [http://statistics.berkeley.edu/sites/ default/files/tech-reports/745.pdf]

31. Bengtsson H, Wirapati P, Speed TP: A single-array preprocessing method for estimating full-resolution raw copy numbers from all Affymetrix genotyping arrays including GenomeWideSNP 5 \& 6. Bioinformatics 2009, 25:17.

32. Ortiz-Estevez M, Aramburu A, Bengtsson H, Neuvial P, Rubio A: CalMaTe: A method and software to improve allele-specific copy number of SNP arrays for downstream segmentation. Bioinformatics 2012, 28:1793-1794.

33. Olshen AB, Bengtsson $H$, Neuvial P, Spellman P, Olshen RA, Seshan VE: Parent-specific copy number in paired tumor-normal studies using circular binary segmentation. Bioinformatics 2011, 27:2038-2046.

34. Carter S, Meyerson M, Getz G: Accurate estimation of homologue-specific DNA concentration-ratios in cancer samples allows long-range haplotyping. [http://hdl.handle.net/10101/npre.2011.6494.1]

35. Development Core Team R: R: A language and environment for statistical computing. R Foundation for Statistical Computing, Vienna, Austria [http://www.R-project.org/]

36. Martin M: Cutadapt removes adapter sequences from high-throughput sequencing reads. EMBnetjournal 2011, 17:1.

37. Li H, Durbin R: Fast and accurate short read alignment with Burrows-Wheeler Transform. Bioinformatics 2009, 25:1754-1760.

38. PICARD. [http://broadinstitute.github.io/picard/]

39. DePristo M, Banks E, Poplin R, Garimella KV, Maguire JR, Hartl C, Philippakis AA, del Angel G, Rivas MA, Hanna M, McKenna A, Fennell TJ, Kernytsky AM, Sivachenko AY, Cibulskis K, Gabriel SB, Altshuler D, Daly MJ: A framework for variation discovery and genotyping using next-generation DNA sequencing data. Nat Genet 2011, 43:491-498.

40. Cibulskis K, Lawrence MS, Carter SL, Sivachenko A, Jaffe D, Sougnez C, Gabriel S, Meyerson M, Lander ES, Getz G: Sensitive detection of somatic point mutations in impure and heterogeneous cancer samples. Nat Biotechnol 2013, 31:3.

41. Ramos AH, Lawrence MS, Pugh TJ, Lichtenstein L, Meyerson M, Getz G Oncotator: cancer variant annotation tool. [http://www.broadinstitute.org/ oncotator/]

42. Ghalanos A, Theuss S: Rsolnp: general non-linear optimization using augmented Lagrange multiplier method. R package version 1.14 [http://www.cran.r-project.org/web/packages/Rsolnp]

43. Ye Y: SOLNP Users Guide, A Nonlinear Optimization Program in MatLab. Stanford University 1989 [web.stanford.edu/ yyye/matlab/manual.ps]

44. Green PJ: Iteratively reweighted least squares for maximum likelihood estimation, and some robuset and resistant alternatives. J R Stat Soc Series B 1984, 46:149-192.

45. Muthukrishnan R: M-estimators in regression models. J Math Res 2010, 2:4.

\section{doi:10.1186/s13059-014-0470-7}

Cite this article as: Lönnstedt et al:: Deciphering clonality in aneuploid breast tumors using SNP array and sequencing data. Genome Biology 2014 15:470. 